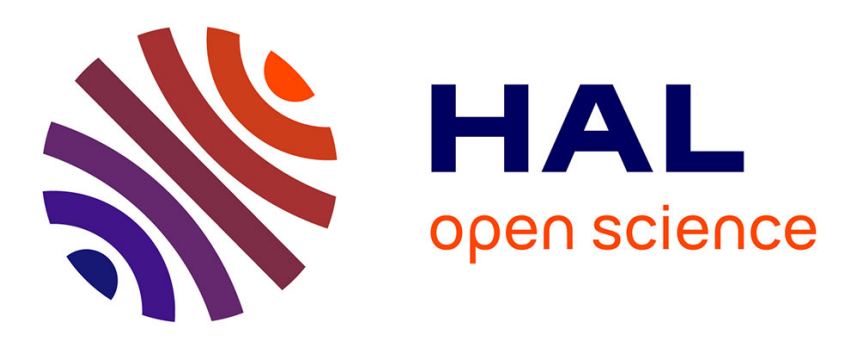

\title{
Piecewise closed-loop equilibria in differential games with regime switching strategies
}

Ngo Van Long, Fabien Prieur, Mabel Tidball, Klarizze Puzon

\section{To cite this version:}

Ngo Van Long, Fabien Prieur, Mabel Tidball, Klarizze Puzon. Piecewise closed-loop equilibria in differential games with regime switching strategies. Journal of Economic Dynamics and Control, 2017, 76, pp.264-284. 10.1016/j.jedc.2017.01.008 . hal-01449990

\section{HAL Id: hal-01449990 https://hal.science/hal-01449990}

Submitted on 30 Jan 2017

HAL is a multi-disciplinary open access archive for the deposit and dissemination of scientific research documents, whether they are published or not. The documents may come from teaching and research institutions in France or abroad, or from public or private research centers.
L'archive ouverte pluridisciplinaire HAL, est destinée au dépôt et à la diffusion de documents scientifiques de niveau recherche, publiés ou non, émanant des établissements d'enseignement et de recherche français ou étrangers, des laboratoires publics ou privés. 


\section{Accepted Manuscript}

Piecewise closed-loop equilibria in differential games with regime switching strategies

Ngo Van Long, Fabien Prieur, Mabel Tidball, Klarizze Puzon

PII:

S0165-1889(17)30016-7

DOI:

10.1016/j.jedc.2017.01.008

Reference:

DYNCON 3391

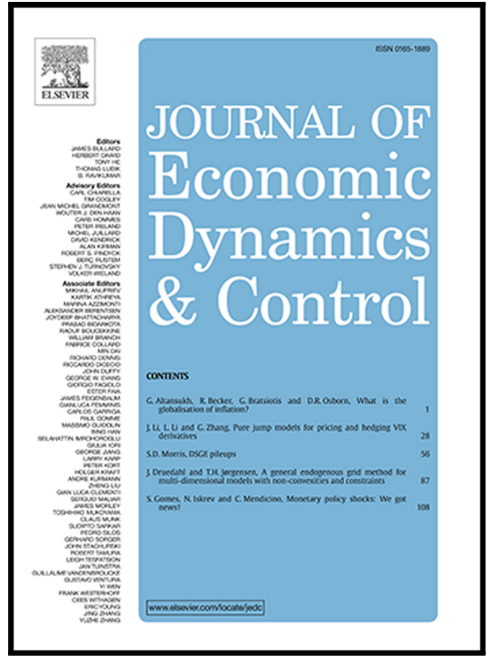

To appear in:

Journal of Economic Dynamics and Control

Received date:

17 September 2015

Revised date:

11 January 2017

Accepted date:

14 January 2017

Please cite this article as: Ngo Van Long, Fabien Prieur, Mabel Tidball, Klarizze Puzon, Piecewise closed-loop equilibria in differential games with regime switching strategies, Journal of Economic Dynamics and Control (2017), doi: 10.1016/j.jedc.2017.01.008

This is a PDF file of an unedited manuscript that has been accepted for publication. As a service to our customers we are providing this early version of the manuscript. The manuscript will undergo copyediting, typesetting, and review of the resulting proof before it is published in its final form. Please note that during the production process errors may be discovered which could affect the content, and all legal disclaimers that apply to the journal pertain. 


\section{Piecewise closed-loop equilibria in} differential games with regime switching strategies*

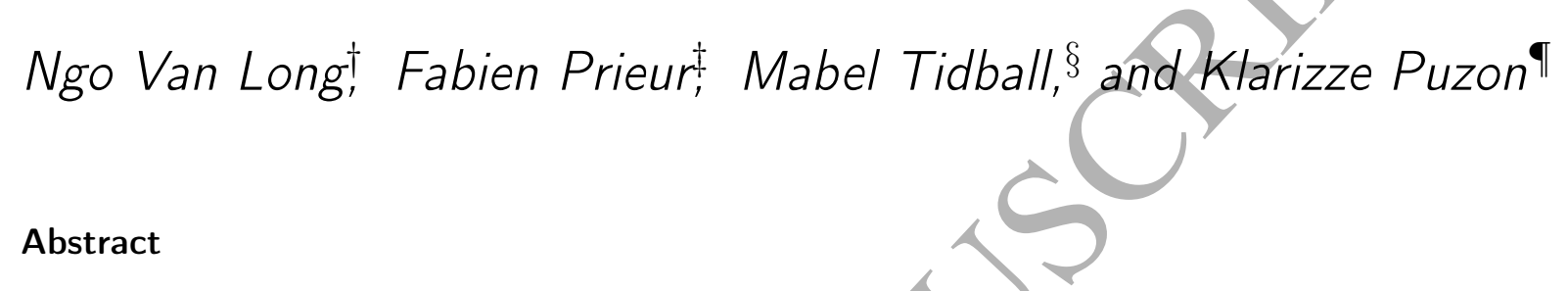

We propose a new methodology exploring piecewise clósed-loop equilibrium strategies in differential games with regime switching actions. We develop a general game with two players. Players choose an action that influences the evolution of a state variable, and decide on the switching time from one regime to another. Compared to the optimal control problem with regime switching, necessary optimality conditions are modified for the first player to switch. When choosing her optimal switching strategy, this player considers the impact of her choice on the other player's actions and consequently on her own payoffs. In order to determine the equilibrium timing of regime changes, we derive conditions that help eliminate candidate equilibrium strategies that do not survive deviations in switching strategies. We then apply this new methodology to an exhaustible resource extraction game.

Key words: differential games; regime switching strategies; technology adoption; non-renewable resources

JEL classification: C61, C73, Q32.

*We thank Raouf Boucekkine, Larry Karp, Santanu Roy, Amos Zemel and participants in conferences and seminars in Annecy, Baton Rouge, Lisbon, Los Angeles and Toulouse. We are also grateful to the Editor, Herbert Dawid, and three anonymous referees for their valuables comments.

${ }^{\dagger}$ Department of Economics, McGill University, 855 Sherbrooke Street West, Montreal, QC H3A 2T7, Canada. E-mail: ngo.long@mcgill.ca

‡UMR EconomiX, University Paris Nanterre, 94000 Nanterre, France. E-mail: fabien.prieur@u-paris10.fr

§INRA-LAMETA, 2 place Viala 34060 Montpellier, France E-mail: tidball@supagro.inra.fr

`LAMETA, University of Montpellier. E-mail: puzon@lameta.univ-montp1 fr 


\section{Introduction}

Several decision making problems in economics concern the timing of switching between alternative and consecutive regimes. Regimes may refer to technological and/or institutional states of the world. For instance, a firm with an initial level of technology may find it optimal to either adopt a new technology or to stick with the old one (Boucekkine et al. 2004). Another example is the decision to phase out existing capital controls in a given economy (Makris, 2001). In all non-trivial problems, switching regime is a decision that involves a trade-off, since adopting a new regime brings with it immediate costs as well as potential future benefits.

There exists a rich literature that deals with endogenous regime shifts modeled as random, for instance poisson-type, processes (see van der Ploeg and de Zeeuw, 2014, and Zemel, 2015, for recent applications to climate change economics). The main difference with this literature is that inducing a regime change is an additional choice variable in the hands of the decision maker; not a risky event one has to cope with. Another distinction is worth doing between two cases: the one where a regime shift is caused by one's rival (e.g., the rival makes a lumpy investment, such as adopting a new harvesting technology), and the one where it is induced by nature itself (e.g., when the carbon stock exceeds a critical threshold level). The focus of our paper is on the former case. The latter case (a natural threshold level) has been investigated by Tahvonen and Withagen (1996), in deterministic setting, and Lemoine and Traeger (2014), in a stochastic enyironment where the policy maker learns about the threshold level by observing the system response in each period.

In this article, we depart from this literature as we consider regime switching strategies in differential games. The game theoretic literature involving regime switching choice is sparse. Early papers on dynamic games of regime change do not involve a stock variable. In these models, the only relevant state of the system is the identity of the players who have adopted the new technology. An example is Reinganum (1981)'s model of technological adoption decisions of two ex ante identical firms. She assumed that firms adopt pre-commitment (open-loop) strategies. That is, it is as if a firm enters a binding commitment on its date of technology switch, knowing the adoption date of the other firm. Reinganum's primary finding is that, with two ex-ante identical firms using open-loop strategies, the equilibrium features diffusion: One firm will innovate first and the other will innovate at a later date. The first player to switch earns higher profits. Fudenberg and Tirole (1985) revisited Reinganum's study by using the concept 
of pre-emption equilibrium. Focusing on Markov perfect equilibrium (MPE) as the solution concept, they noted that the second player to switch may try to preempt its rival and become the first to adopt. At the preemption equilibrium, the first player to switch advantage vanishes (see Long, 2010, for a survey of this literature).

A second strand of literature pertains to the strategic interaction of agents in relation to the dynamics of a given stock. For instance, Tornell (1997) presented a model that explores the relationship between economic growth and institutional change. Infinitelylived agents solve a differential game that drives the changes in property-rights regimes for the economy's capital stock, e.g. common property versus private property. It was shown that a potential equilibrium of the game involves multiple switching between regimes. However, only the symmetric equilibrium was considered, such that the players always choose to switch at the same instant. Consequently, the question of the timing between switching times was not addressed. In addition, even though Tornell explicitly defined the MPE for the class of differential games with regime switching, he does not provide a general modeling of switching strategies. One can also mention the related analysis by Boucekkine et al. (2011). They analyzed the trade-off between environmental quality and economic performance using a simple two-player differential game where players may switch to a cleaner but less productive technology. But they paid attention to the open-loop Nash equilibrium only while our view is that commitment requirements are simply too strong to model switching strategies as open-loop strategies.

Undoubtedly, accounting for the existence of some sort of feedback effect in players' switching strategies operating through the state of the system is a challenging task. In particular, it does not seem straightforward at first glance to extend the standard definition of markovian rules (whereby strategies precisely depend on the state of the system) to this specific class of discrete decisions. To our knowledge, there seems to be no existing study which formally adresses this issue. This is where the first theoretical contribution of this paper lies. We develop a general differential game with two players having two kinds of strategies. First, players have to choose at each point in time an action that influences the evolution of a state variable. Second, they may decide on the timing of switching between alternative and consecutive regimes that differ both in terms of the payoff function and the state equation. İn this setting, we define a new equilibrium concept, referred to as the piecewise closed-loop Nash equilibrium (PCNE hereafter), which is the natural adaptation of the MPE when timing decisions are part 
of the players' strategy profile. Formally, this boils down to expressing a player's timing strategy as a function of the state of the system, which is described by the regime that is in current operation, and the level of the stock variable at which the player's regime switching problem starts.

For any possible timing, we characterize the necessary optimality conditions for switching times, both for interior and corner solutions. One interesting finding is that, compared to the necessary conditions characterizing optimal switching in the standard (one-player) optimal control problem or in game with open-loop information structure, we find that the necessary optimal switching conditions are substantially modified for the player who finds it optimal to move first. Indeed, when choosing the optimal date and level of the state variable for switching, this player must take into account that (i) her decision will influence the other player's equilibrium switching strategy in the subgame that follows, and (ii) the other player's switching time will impact on her own welfare. Depending on the particular economic problem at hand, the interaction through switching times may provide an incentive to either postpone or expedite regime switching. Another important issue is how to determine the equilibrium switching sequence in the PCNE. We resolve this issue by providing conditions that help eliminate candidate switching sequences that do not survive deviations in switching strategies.

The second contribution of the paper is the application of this new game theoretic material to study a model of management of an exhaustible resource. To date, there are only a few papers that have studied the relationship between natural resource exploitation and the timing of technology adoption. Using a finite horizon two-stage optimal control problem, Amit (1986) explored the case of a petroleum producer who considers switching from a primary to a secondary recovery process. He observed that a technological switch occurs if the desired extraction rate is greater than the one that can be obtained by the natural drive, or when the desired final output is more than what can be obtained/using the primary process. In a recent paper, Valente (2011) analyzed a two-phase endogenous growth model which concerns a switch from an exhaustible resource input into a backstop technology. He showed that adoption of new technology implies a sudden fall in consumption, but an increase in the growth rate. ${ }^{1}$

We extend this literature by developing a simple differential game of resource extraction with technology adoption. In our setting, two players extract an exhaustible

\footnotetext{
${ }^{1}$ In the same vein, Boucekkine et al. (2013) explored a general control problem with both technological and ecological regime switches. They applied it to address the issue of optimal resource extraction under ecological irreversibility, and with the possibility to adopt a backstop technology.
} 
resource for consumption purposes. By incurring a lumpy cost, they can adopt a more efficient extraction technology. So not only do players choose their extractions rates, they also decide whether to adopt the new technology and when. In the application, we first explicitly characterize strategies at the PCNE where both players switch to the new technology in finite time. We especially focus on the specific economic trade-offs related to the new type of regime switching strategies. Then, we investigate the impact of these strategies on both the extraction rates and the timing of adoption of new technologies. Particular emphasis is on the effect of closed-loop strategic interaction on the first player to switch's strategy (as compared to the single-agent case). We show that since adoption of the second player to switch is costly for the first player, the latter strategically chooses his date of adoption so that he induces the former to postpone her adoption. Finally, we deal with the issue of what timing should ultimately arise at the equilibrium by putting forward some conditions under which none of the players have an incentive to deviate from a specified timing.

The paper is organized as follows. Section 2 presents the details of the general setup. Section 3 deals with the theory and characterizes optimality conditions specific to regime switching strategies. In Section 4 , we study the resource extraction game to illustrate how our methodology works in a simple application. Section 5 is devoted to a discussion of a numerical example and also reviews other potentially interesting and more involved economic applications of our theory. Finally Section 6 concludes.

\section{The general problem}

We consider a two-player differential game in which the instantaneous payoff of each player and the differential equation describing the stock dynamics depend on what regime the system is in. There are a finite number of regimes, indexed by $s$, and we assume that under certain conditions, the players are able to take action (at some cost) to affect a change of regime. Let $\mathcal{S}$ be the set of regimes. For simplicity, we assume that each player can make a regime switch only once. ${ }^{2}$ This implies that regime changes are irreversible, i.e., switching back is not allowed. In this case, there are four possible regimes and the set $\mathcal{S}$ is simply: $\mathcal{S} \equiv\{11,12,21,22\}$.

We assume that the system is initially in regime 11 . Player 1 can take a "regime

\footnotetext{
2 The assumption is made for convenience. It would be easy to generalize the analysis to situations where the players can choose any (finite) number of switching dates by emulating Makris (2001)'s approach (who considers control problems with many switching times) and combining it with ours.
} 
switching action" to switch the system from regime 11 to regime 21, if the other player has not made a switch. The first number in any regime index indicates player 1's moves. The second refers to player 2 . Once the system is in regime 21 , only player 2 can take a regime switching action, and this leads the system to regime 22. From regime 11, player 2 can switch to regime 12 (if player 1 has not made a switch). From regime 12, only player 1 can make a regime change, and this switches the system to regime 22 . If the system is in regime 11 and both players take regime change action simultaneously, the regime will be switched to 22. Finally, the system may remain in 11 forever if neither agent takes a regime change action. Let $\mathcal{S}_{i}$ be the subset of $\mathcal{S}$ in which player $i$ can make a regime change. Then $\mathcal{S}_{1}=\{11,12\}$ and $\mathcal{S}_{2}=\{11,21\}$.

The state variable $x$ is a continuous function for all $t$ and could be in any space $\mathbb{R}_{+}^{m}, 1 \leq m \leq M$. At each instant, each player chooses an action $u_{i}$, with $u_{i} \in \mathbb{R}^{n_{i}}$, $1 \leq n_{i} \leq N_{i}<\infty$, that affects the evolution of $x$. To simplify the exposition, we set $m=1$ and $n_{i}=n$ for $i=1,2$. The instantaneous payoff to player $i$ at time $t$ when the system is in regime $s$ is

$$
F_{i}^{s}\left(u_{i}(t), u_{-i}(t), x(t)\right)
$$

If player $i$ takes a regime change action at time $t_{i} \in \mathbb{R}_{+}$, a lumpy cost $\Omega_{i}\left(t_{i}, x\left(t_{i}\right)\right)$ is incurred. Then, if for example $0<t_{1} \leqslant t_{2}<\infty$, player 1's total payoff is

$$
\begin{aligned}
& \int_{0}^{t_{1}} F_{1}^{11}\left(u_{1}, u_{2}, x\right) e^{-\rho t} d t+\int_{t_{1}}^{t_{2}} F_{1}^{21}\left(u_{1}, u_{2}, x\right) e^{-\rho t} d t \\
& +\int_{t_{2}}^{\infty} F_{1}^{22}\left(u_{1}, u_{2}, x\right) e^{-\rho t} d t-\Omega_{1}\left(t_{1}, x\left(t_{1}\right)\right),
\end{aligned}
$$

with $\rho$ the discount rate. The differential equation describing the evolution of the state variable $x$ in regime $s$ is

$$
\dot{x}=f^{s}\left(u_{1}, u_{2}, x\right) .
$$

Let us now explain what is meant by the expression "strategy profile" in this setting in which each player has two types of controls. The set of controls is given by $\mathcal{C}_{i}=$ $\left\{u_{i}, t_{i}\right\}$. A strategy consists of an action policy, describing the actions undertaken by each player at every possible state of the system, $(x, s) \in \mathbb{R}_{+} \times S$, and a switching rule that represents the decision to switch for some relevant level of the state variable. Again, for the sake of exposure, we restrict attention to those strategies that are not timedependent. This requires that the function $\Omega_{i}\left(t_{i}, x\left(t_{i}\right)\right)$ takes the form $e^{-\rho t_{i}} \omega_{i}\left(x\left(t_{i}\right)\right)$.

Player $i$ 's action policy is a mapping $\Phi_{i}$ from the state space $\mathbb{R}_{+} \times \mathcal{S}$ to the set $\mathbb{R}^{n}$. 
This is the standard definition of feedback rules for continuous controls. This definition is not directly transposable to switching strategies, which correspond to a specific type of discrete controls. However, if one wants to introduce a link between these strategies and the state of the system, then it is possible to proceed as follows.

Suppose player 1 thinks that if player 2 finds herself in regime 21 at date $t$, with $x(t)$ (which implies that he switched at an earlier date $t_{1} \leq t$ ), she will make a switch at a date $t_{2} \geq t$. Then player 1 should think that the interval of time between his own switching date $t_{1}$ and the switching date $t_{2}$ is a function not of any level of the stock, but of the value of the stock at which player 1's regime change takes place, i.e., at $x\left(t_{1}\right)=x_{1}$. More generally, we can define the timing strategy of player $i$, given that $s \in \mathcal{S}_{i}$, as a mapping $\theta_{i}$ from $\mathbb{R}_{+} \times \mathcal{S}$ to $\mathbb{R}_{+} \cup\{\infty\}$. For instance, from the state $\left(x_{1}, 21\right), \theta_{2}\left(x_{1}, 21\right)$ is the length of time that must elapse before player 2 takes her regime switching action, i.e, the duration of regime 21. If $\theta_{2}\left(\hat{\left.x_{1}, 21\right)}=\infty\right.$, then it means that she does not want to switch at all from regime 21 .

One may note that the $\theta_{i}$ s are not markovian in the usual sense as they are not defined over any possible level of the stock variable. Indeed, modeling timing decisions as standard feedback strategies is problematic. It implies that the time to go before the next switch of player $i$ is a function of the stock $x(t): \theta_{i}(x(t), s)$, for all $t \geq t_{-i} \geqq 0$ $\left(t_{-i}\right.$ is either the initial time or the switching time of the other player). Assume that the regime switch finally occurs at $t_{i}$ and for a level $x_{i}$. In order to determine $\theta_{i}$, one needs to know the evolution of $x$ on the interval $\left[t, t_{i}\right]$. But this depends on the action policies, $\Phi_{i}$, of both players, and in particular on that of player $-i$, which is obviously not controlled by player $i$. This in turn means that $x$ cannot be part of player $i$ 's switching strategy as otherwise it wouldn't be robust to deviations. Put differently, we would then face the problem that the rule itself, i.e., the function $\theta_{i}$, changes as a result of a deviation in $\Phi_{-i}$ during some interval of time in between $t$ and $t_{i}{ }^{3}$

Switching strategies nevertheless feature a dependence on a specific value of the stock, the one at which each player's switching problem begins, and for this reason, they can be labelled as piecewise closed-loop strategies. The corresponding new equilibrium concept we then introduce is referred to as the piecewise closed-loop (Nash) equilibrium (see/e.g., Davis, 1993, and Haurie and Moresino, 2001, for related works). The key point is that such a formulation allows us to account for a new kind of feedback effect in players' switching strategies channeling through the state of the system. Our approach

\footnotetext{
3 This is not the same thing as saying that the decision can be revised in response to changes in the stock variable, induced by a deviation.
} 
obviously differs from what one would obtain with an open-loop information structure whereby each player chooses her switching time taking the one of the other as given. This leads to the following definition.

Definition 1. - A strategy vector of player $i$ (as guessed by player -i) is a pair $\psi_{i} \equiv\left(\Phi_{i}, \theta_{i}\right), i=1,2$.

- A strategy profile is a pair of strategy vectors, $\left(\psi_{1}, \psi_{2}\right)$.

- A strategy profile $\left(\psi_{1}^{*}, \psi_{2}^{*}\right)$ is called a piecewise closed-loop (Nash) equilibrium (PCNE), if given that player $i$ uses the strategy vector $\psi_{i}^{*}$, the payoff of player $j$, starting from any state $(x, s) \in \mathbb{R}_{+} \times \mathcal{S}$, is maximized by using the strategy vector $\psi_{j}^{*}$, where $i, j=1,2$.

In order to determine a PCNE of this game, a natural way to proceed, for any particular timing, is to solve (i) the problem corresponding to each subgame in the action policies, taking as given the switching times $\left(t_{1}, t_{2}\right),{ }^{4}$ and (ii) the switching problem of each player in the switching rules, for a particular profile of action strategies $\left(\Phi_{1}, \Phi_{2}\right)$. For that purpose, we shall extend the methodology originally developed by Tomiyama (1985) and Amit (1986) to solve finite horizon two-stage optimal control problems (for infinite horizon problems, see Makris, 2001). In order to apply this methodology, we need to impose:

Assumption 1. The functions $F_{i}^{s}($.$) and f^{s}($.$) , for any s \in \mathcal{S}$, belong to the class $C^{1}$. Moreover, the sub-game obtained by restricting the general problem to any regime $s$ satisfies the Arrow-Kurz sufficiency conditions.

This assumption ensures that our problem is well-behaved and we manipulate smooth enough functions.

Before going to the theoretical analysis, we would like to emphasize that the topic of the paper is all about regime switching strategies in differential games. This means that the core of the analysis is entirely devoted to a presentation of the optimality conditions associated with these strategies and a discussion on the impact of this new source of interaction on players' behaviors. We thus abstract from other issues that typically arise in differential games, like existence and uniqueness of the equilibrium

\footnotetext{
${ }^{4}$ As illustrated by the decomposition above, if the equilibrium timing is such that $0 \leqq t_{1} \leqq t_{2} \leqq \infty$, then there are three sub-games to be considered, each being associated with a particular regime. Indeed, for the timing considered, the sequence of regimes is: 11, 21 and 22.
} 
by assuming that a solution exists and focusing on the novel part of the problem. ${ }^{5}$ The next section presents the set of necessary optimality conditions that characterize a PCNE of the differential game with regime switching strategies.

\section{Optimality conditions for switching strategies}

The analysis is conducted for a particular timing: $0 \leqq t_{1} \leqq t_{2} \leqq \infty$ Necessary optimality conditions for the other general timing, $0 \leqq t_{2} \leqq t_{1} \leqq \infty$, can easily be derived by symmetry. First, we state and interpret optimality conditions for an interior solution (a solution with $t_{i}$ positive and finite and $t_{1} \neq t_{2}$ ), which allows us to highlight the impact of the interaction through switching strategies on the solution. Next, we want to know whether a player has an incentive to deviate from the timing considered. For that purpose, corner solutions are carefully studied.

\subsection{Interior solution}

Assume that there exists a solution $\left(u_{1}^{*}(t), u_{2}^{*}(t), x^{*}(t)\right)$ to the differential game defined above and for given $\left(t_{1}, t_{2}\right)$. In any regimes, Player $i$ 's present value Hamiltonian, $H_{i}^{s}=$ $F_{i}^{s}\left(u_{i}, \Phi_{-i}(x, s), x\right) e^{-\rho t}+\lambda_{i}^{s} f^{s}\left(u_{i}, \Phi_{-i}(x, s), x\right)$ with $\lambda_{i}^{s}$ the co-state variable, evaluated at this solution is denoted by $H_{i}^{s *}$ and we refer to $\theta_{2}^{\prime}$ as the derivative w.r.t the state variable $x$. Our first theorem states the necessary optimality conditions related to the switching strategies at the interior solution, if it exists. All the proofs are displayed in the Appendix A.1.

Theorem 1. The necessary optimality conditions for the existence of a PCNE featuring the timing $0<\widehat{t_{1}}<t_{2}<\infty$ are:

- For player 2:

$$
\begin{aligned}
& H_{2}^{21 *}\left(t_{2}\right)-\frac{\partial \Omega_{2}\left(t_{2}, x^{*}\left(t_{2}\right)\right)}{\partial t_{2}}=H_{2}^{22 *}\left(t_{2}\right) \\
& \lambda_{2}^{21}\left(t_{2}\right)+\frac{\partial \Omega_{2}\left(t_{2}, x^{*}\left(t_{2}\right)\right)}{\partial x_{2}}=\lambda_{2}^{22}\left(t_{2}\right) .
\end{aligned}
$$

\footnotetext{
${ }^{5}$ In the literature on differential games, general theorems on existence are not available, except for linear quadratic games (e.g., Haurie et al., 2012), or some specific class of stochastic capital accumulation games (e.g. Dutta and Sundaram, 1993, and Amir, 1996). Even for linear quadratic games, uniqueness cannot be guaranteed. See Dockner et al. (1996) for examples of non-uniqueness.
} 
- For player 1:

$$
\begin{aligned}
& H_{1}^{11 *}\left(t_{1}\right)-\frac{\partial \Omega_{1}\left(t_{1}, x^{*}\left(t_{1}\right)\right)}{\partial t_{1}}=H_{1}^{21 *}\left(t_{1}\right)-\left[H_{1}^{21 *}\left(t_{2}\right)-H_{1}^{22 *}\left(t_{2}\right)\right] \\
& \lambda_{1}^{11}\left(t_{1}\right)+\frac{\partial \Omega_{1}\left(t_{1}, x^{*}\left(t_{1}\right)\right)}{\partial x_{1}}=\lambda_{1}^{21}\left(t_{1}\right)+\theta_{2}^{\prime}\left(x^{*}\left(t_{1}\right), 21\right)\left[H_{1}^{21 *}\left(t_{2}\right)-H_{1}^{22 *}\left(t_{2}\right)\right],
\end{aligned}
$$

To understand these switching conditions for an interior solution, let us focus on the difference between the optimality conditions of the first player to switch (player 1) and the second (player 2) for the particular timing considered. Player 2's conditions (1) are similar to the ones derived in multi-stage optimal control literature. Condition (1a) states that it is optimal to switch from the penultimate to the final regime when the marginal gain of delaying the switch, given by the difference $H_{2}^{21 *}()-.H_{2}^{22 *}($.$) ,$ is equal to the marginal cost of switching, $\frac{\partial \Omega_{2}\left(t_{2}, x^{*}\left(t_{2}\right)\right)}{\partial t_{2}}$. Condition (1b) equalizes the marginal benefit from an extra unit of the state variable $x\left(t_{2}\right)$ with the corresponding marginal cost. It basically says that the value of the co-state, when approached from the intermediate regime, plus the incremental switching cost must just equal the value of the co-state, approached from the final regime. Hence, as long as a player finds it optimal to be the second player to switch, her optimality conditions are similar to the standard switching conditions of an optimal control problem.

The novel part of Theorem 1 stems from the problem faced by the player who opts to switch first. Indeed, player 1's optimality conditions are modified (compared to the single agent framework). The first condition (2a) implies that player 1 cares about changes in his situation induced by the switch of player 2. Player 1 decides on his optimal switching time by equalizing the marginal gain of delaying the switch, which is given by the difference $H_{1}^{11 *}()-.H_{1}^{21 *}($.$) to the marginal switching cost, \frac{\partial \Omega_{1}\left(t_{1}, x^{*}\left(t_{1}\right)\right)}{\partial t_{1}}-$ $\left[H_{1}^{21 *}\left(t_{2}\right)-H_{1}^{22 *}\left(t_{2}\right)\right]$. The extra-term $\left[H_{1}^{21 *}\left(t_{2}\right)-H_{1}^{22 *}\left(t_{2}\right)\right]$ is the marginal impact of player 2's switch on player 1 . So, player 1 anticipates the impact of player 2's switch on his payoff. Depending on the nature of the problem, the additional term can either be positive or hegative. The second optimality condition $(2 \mathrm{~b})$ is also modified. The cost of a marginal increase in $x$ at $t_{1}$ now includes an extra-term: $\theta_{2}^{\prime}\left(x^{*}\left(t_{1}\right), 21\right)\left[H_{1}^{21 *}\left(t_{2}\right)-\right.$ $\left.H_{1}^{22 *}\left(t_{2}\right)\right]$. This term reflects the fact that player 1 takes into account his influence on player 2's timing strategy, through the level of the state variable at the switching time $x^{*}\left(t_{1}\right)$. Put differently, player 1 knows that modifying $x^{*}\left(t_{1}\right)$ is a means to delay or accelerate player 2's regime switching. In sum, the modified switching conditions of player 1 illustrate the existence of a two-way interaction through switching strategies. 
A couple of comments are in order here. First, in (2), the term $\theta_{2}^{\prime}\left(x^{*}\left(t_{1}\right), 21\right)$ may look like a kind of Stackelberg-leadership consideration: Player 1 knows the function $\theta_{2}^{* \prime}\left(x^{*}\left(t_{1}\right), 21\right)$, and hence he knows that when he chooses $t_{1}$ and the level $x^{*}\left(t_{1}\right)$ he is indirectly influencing $t_{2}$. But this is not really Stackelberg leadership in a global sense. The situation is just like any standard game tree with sequential moves. If a player moves first, he knows how the second player to switch will move at each subgame that follows, and therefore he will take that into account in choosing which subgame he is going to induce.

Second, the methodology used here relies extensively on the tools developed by the multi-stage control theory. Beyond the difference in terms of optimality conditions discussed above, there is another crucial difference between a three-stage optimal control problem and our three-stage differential game. Indeed in the former problem, the two regime switches are triggered by a single agent and each is associated with optimality conditions similar to (1). Put differently, a single agent would choose two different switching instants $t_{1}, t_{2} \in(0, \infty)$ and the corresponding optimal values for the state variable $x^{*}\left(t_{1}\right)$ and $x^{*}\left(t_{2}\right)$. In the game version and for the timing considered here, player 1 - who is supposed to switch first $\leftarrow$ has no such optimality conditions at player 2 ' switching time $t_{2}$. He's neither the one that has to choose the switching time. Nor, can he optimally adjust the level of the state variable at this instant. Similarly, there are no optimality conditions for player 2 at $t_{1}$, the instant when player 1 solves his own switching problem (see, for more details, pages 33-36 of the Appendix A.1). However, as mentioned just above, for this particular sequence of moves, player 1 can indirectly influence player 2's switching decision whereas player 2 cannot.

\subsection{Corner solutions}

Still for the same timing, we now examine the conditions for one player to choose a corner strategy.

Theorem 2. 1. Suppose player 1 switches at some instant $t_{1} \in(0, \infty)$.

- Necessary conditions for player 2 to choose a corner solution with immediate switching, i.e., $t_{2}=t_{1}$ (instead of $t_{2}>t_{1}$ ) are (1b), and

$$
H_{2}^{21 *}\left(t_{2}\right)-\frac{\partial \Omega_{2}\left(t_{2}, x^{*}\left(t_{2}\right)\right)}{\partial t_{2}} \leq H_{2}^{22 *}\left(t_{2}\right) \text { if } t_{1}=t_{2}
$$


2. Suppose player 2's switching problem has an interior solution $t_{2}$.

- Necessary conditions for player 1 to choose a corner solution with immediate switching $0=t_{1}$ are (2b), and

$$
H_{1}^{11 *}\left(t_{1}\right)-\frac{\partial \Omega_{1}\left(t_{1}, x^{*}\left(t_{1}\right)\right)}{\partial t_{1}} \leq H_{1}^{21^{*}}\left(t_{1}\right)-\left[H_{1}^{21 *}\left(t_{2}\right)-H_{1}^{22 *}\left(t_{2}\right)\right] \text { if } 0=t_{1}<t_{2}
$$

- Necessary conditions for player 1 to choose a corner solution of the never switching type $t_{1}=t_{2}$ are (2b), and

$$
H_{1}^{11 *}\left(t_{1}\right)-\frac{\partial \Omega_{1}\left(t_{1}, x^{*}\left(t_{1}\right)\right)}{\partial t_{1}} \geq H_{1}^{21^{*}}\left(t_{1}\right)-\left[H_{1}^{21 *}\left(t_{2}\right)-H_{1}^{22 *}\left(t_{2}\right)\right], \text { if } t_{1}=t_{2}
$$

The corner solution $t_{1}=0$ and corresponding necessary conditions have already been discussed in literature. For $t_{1}=0$, it must be the case that player 1 wants to escape from regime 11 as soon as possible. According to condition (4), this happens when a delay in switching yields a marginal gain that is not greater than the marginal loss of foregoing for an instant the benefit of the new regime. Of further interest is the interpretation of players' "corner solutions" $t_{1}=t_{2}$. Quotes are needed because those solutions actually correspond to artificial situations where the timing is (pre)specified (here $t_{1} \leqq t_{2}$ ). The conditions for them to occur deserve much attention since they provide a clear way to check if a candidate equilibrium in switching strategies is robust to deviations. As an illustration, consider player 1's problem. Conditional on player 1 being the first to make a switch and on player 2 being the second, we can derive a necessary condition for $t_{1}$ to be at the corner $t_{1}=t_{2}$. If this condition (5) is satisfied, which means that at $t_{2}$ a delay in switching yields a marginal gain that is at least as high as the marginal loss of foregoing for an instant the benefit of the new regime, then we suspect that, when we remove the artificial requirement that $t_{1} \leqq t_{2}$, there will be an incentive for/player 1 to choose to make a regime switch in second place. In such a case, a candidate solution with $t_{1} \leqq t_{2}$ does not survive the incentive for player 1 to deviate from it. In other words, condition (5) is necessary for the timing not to be robust to deviations in player 1's switching strategy. The same reasoning applies to player 2's corner solution $t_{2}=t_{1}$.

Of course, the timing is not fixed in our differential game with regime switching strategies and the most important task is precisely to determine what will be the timing at the equilibrium, or under which conditions a particular timing will occur. The 
analysis of situations where one player may have an incentive to deviate is of crucial importance to address this non-trivial issue. Indeed, it should allow us to understand which timing, between $0 \leqq t_{1}<t_{2} \leqq \infty$ and $0 \leqq t_{2}<t_{1} \leqq \infty$, is consistent with the PCNE requirement.

Let us conclude this section with a brief overview of the other possible combinations between $t_{1}$ and $t_{2}$. First, note that there is no counterpart to the necessary conditions (3)-(5) for the corner solution $t_{2}=\infty$ (see the discussion in Makris, 2001, page 1941). However, the inequality

$$
H_{2}^{21 *}\left(t_{2}\right)-\frac{\partial \Omega_{2}\left(t_{2}, x^{*}\left(t_{2}\right)\right)}{\partial t_{2}}>H_{2}^{22 *}\left(t_{2}\right) \text { for all } t_{1} \leq t_{2}<\infty
$$

is sufficient to establish that player 2 will never find it optimal to switch regime. Next, it is highly unlikely that heterogeneous players decide on the same switching time. So, generically, we do not expect the timing $0<t_{1}=t_{2}<\infty$ to be a PCNE candidate when players have different costs or preferences. But, it is quite easy to derive the optimality conditions if that timing is an equilibrium outcome for some non-generic cases. ${ }^{6}$

The next section is devoted to an application of the theory to an exhaustible resource problem. Our purpose is to illustratehow the reasoning above works in a simple example from which we can extract analytical results.

\section{A resource extraction game with technology adoption}

We consider a differential game of extraction of a non-renewable resource. In the related literature (for extensive surveys on dynamic games in resource economics, refer to Long, 2010, 2011), it is generally argued that the presence of rivalry among multiple agents tends to result in inefficient outcomes, e.g. overextraction of natural resources. Another common feature of the frameworks developed in this literature is the assumption that players cannot adopt new technology that will improve their extraction efficiency. It

\footnotetext{
${ }^{6}$ Suppose that it is optimal for the two players to switch at the same date $t_{1}=t_{2} \in(0, \infty)$, for the same level of the state $x^{*}(t)=x^{*}\left(t_{1}\right)=x^{*}\left(t_{2}\right)$, then the following conditions must hold, for $i=1,2$ :

$$
\begin{aligned}
& H_{i}^{11 *}(t)-\frac{\partial \Omega_{i}\left(t, x^{*}(t)\right)}{\partial t_{i}}=H_{i}^{22 *}(t) \\
& \lambda_{i}^{11}(t)+\frac{\partial \Omega_{i}\left(t, x^{*}(t)\right)}{\partial x_{i}}=\lambda_{i}^{22}(t) .
\end{aligned}
$$

Finally, conditions corresponding to the cases $t_{1}=t_{2}=0$ and $t_{1}=t_{2}=\infty$ can easily be derived from the material presented above.
} 
is usually assumed that consumption is a fixed fraction of the extraction level. In this section, we relax this assumption and consider the possibility of technological adoption among players. That is, players not only choose their consumption, but they also decide when to adopt the more efficient extraction technology. This consideration represents another contribution of this paper.

Our resource extraction game comprises $I=2$ players. Let $u_{i}(t)$ denote the consumption rate of player $i, i=1,2$, at time $t \geq 0$. Meanwhile, let $e_{i}(t)$ be player $i$ 's extraction rate from the resource at time $t \geq 0$. Extraction is converted into consumption according to the following technology: $\gamma_{i} u_{i}(t)=e_{i}(t)$, where $\gamma_{i}^{-1}$ is a positive number that reflects a player's degree of efficiency in transforming the extracted natural resource into a consumption good.

Two production technologies, described only by the parameter $\gamma_{i}$, are available to player $i$ from $t=0$. Because players' technological menus may differ, one needs to introduce a specific index for the player's actual technology. It is assumed that player 1 starts with technology $l=1$ and has to decide: (i) whether he switches to technology $l=2$, and (ii) when. The state of technology of the other player, 2, is labelled as $k$ and a technological regime is represented by $s=l k$, with $l, k=1,2$. For each player $i$, the ranking between the parameters satisfies: $\gamma_{i}^{1}>\gamma_{i}^{2}$, which means that the second new technology is more efficient than the old one. A possible indicator of technological gain for player $i$ from adoption is the ratio $\frac{\gamma_{i}^{2}}{\gamma_{i}^{1}} \in(0,1)$, such that the smaller is the ratio, the higher is the gain.

Let $x(t)$ be the stock of the exhaustible resource, with the initial stock $x_{0}$ given. As in section $2, t_{1}$ and $t_{2}$ are the switching times. Suppose $0<t_{1}<t_{2}$, then the evolution of the stock is given by the following differential equation:

$$
\dot{x}=\left\{\begin{array}{l}
-\gamma_{1}^{1} u_{1}-\gamma_{2}^{1} u_{2} \text { if } t \in\left[0, t_{1}\right) \\
-\gamma_{1}^{2} u_{1}-\gamma_{2}^{1} u_{2} \text { if } t \in\left[t_{1}, t_{2}\right) \\
-\gamma_{1}^{2} u_{1}-\gamma_{2}^{2} u_{2} \text { if } t \in\left[t_{2}, \infty\right)
\end{array}\right.
$$

At the switching time, if any, player $i$ incurs a cost that is defined in terms of the level of the state variable at which the adoption occurs, $x\left(t_{i}\right)=x_{i}$. Let $\omega_{i}\left(x\left(t_{i}\right)\right)$ be this cost, with $\omega_{i}^{\prime}() \geq$.0 . It takes the form: $\omega_{i}\left(x_{i}\right)=\chi_{i}+\beta_{i} x_{i}$, where $\chi_{i} \geq 0, \beta_{i} \geq 0$, and $\chi_{i}$ is the fixed cost related to technology investment. These may include initial outlay for machinery, etc. On the other hand, $\beta_{i}$ represents the sensitivity of adoption cost to the level of the exhaustible resource at the instant of switch. Our assumption implies 
that the cost of adopting new technology is increasing in $x_{i}$. This assumption conveys the idea that the lower the level of the (remaining) stock of resource, the lower the cost of adopting the new technology. It could reflect the fact that scientific progress on installation of resource-saving technology is continually made as the scarcity becomes more acute. The direct switching cost is discounted at rate $\rho$. As seen from the initial period, if a switch occurs at $t_{i}$, the discounted cost amounts to $e^{-\rho t_{i}} \omega_{i}\left(x_{i}\right)$ (this is our $\Omega_{i}\left(t_{i}, x\left(t_{i}\right)\right)$ of Section 2$)$. Finally, each player's gross utility function depends on her consumption only and takes the logarithmic form: $F\left(u_{i}, u_{-i}, x\right)=\ln \left(u_{i}\right)$.

Hereafter, our aim is to apply the methodology developed in Section 3 to this simple problem. We start by explicitly characterizing both extraction and switching strategies at the PCNE with $0<t_{1}<t_{2}<\infty$. Then, in the wake of the discussion following Theorem 1, we illustrate the impact of strategic interaction on the decision to adopt the new technology. Finally, we present the conditions under which neither the players want to deviate from the timing considered, nor they have an incentive to adopt a corner strategy. Note that in the subsequent analysis, we pay attention only to the class of linear feedback strategies for extraction rates. 7 Moreover, we shall denote $x^{*}\left(t_{i}\right)=x_{i}^{*}$, the level of the stock of resource at which player $i$ decides to switch at the PCNE and call it the switching point. All the proofs are relegated in Appendix B.

\subsection{PCNE with $0<t_{1}<t_{2}<\infty$}

The general game - defined as a sequence of three subgames 11, then 21 , and finally 22 - is solved backward. Let us put aside the issue of the existence of the PCNE for the moment (this analysis is postponed to the very end of Section 4.3), and focus on its characteristics assuming that it exists. In the remainder of the analysis, we need to define the function $\zeta\left(x_{1} ; x_{2}^{*}\right)$ as follows

$$
\zeta\left(x_{1} ; x_{2}^{*}\right)=1-\frac{e^{-\rho \theta_{2}\left(x_{1}, 21\right)}}{2} \ln \left(1-\rho \beta_{2} x_{2}^{*}\right)-\beta_{1}\left(\Gamma\left(x_{2}^{*}\right)+\rho x_{1}\right) \text {. }
$$

For the problem to be well-defined, hereafter we assume that $\zeta\left(x_{0} ; x_{2}^{*}\right)>0$. In order to avoid unnecessary discussion on technical grounds, we also require that $x_{0}<$

7 To echo footnote 6 , it is well-known that there may exist other feedback, non-linear, equilibria in linear-quadratic games (see Dutta and Sundaram, 1993). If we are aware that different types of extraction strategies may lead to a different timing at the PCNE, assessing the issue of uniqueness is beyond the scope of the paper. 
$\min \left\{\left(\rho \beta_{1}\right)^{-1},\left(\rho \beta_{2}\right)^{-1}\right\} .{ }^{8}$ Then we can establish that:

Proposition 1. The PCNE with $0<t_{1}<t_{2}<\infty$ has the following features:

- In regime 22: extraction rates are given by

$$
\gamma_{1}^{2} \Phi_{1}(x, 22)=\gamma_{2}^{2} \Phi_{2}(x, 22)=\rho x
$$

- In regime 21: extraction rates are defined by

$$
\gamma_{1}^{2} \Phi_{1}(x, 21)=\gamma_{2}^{1} \Phi_{2}(x, 21)=\Gamma\left(x_{2}^{*}\right)+\rho x \text { with } \Gamma\left(x_{2}^{*}\right)=\frac{\rho^{2} \beta_{2}\left(x_{2}^{*}\right)^{2}}{1-\beta_{2} \rho x_{2}^{*}} .
$$

There exists a unique switching point, $x_{2}^{*}$, for player 2 , that solves

$$
\rho \omega_{2}\left(x_{2}^{*}\right)=\ln \left(1-\beta_{2} \rho x_{2}^{*}\right)-\ln \left(\frac{\gamma_{2}^{2}}{\gamma_{2}^{1}}\right) .
$$

The time-to-go (before switching) strategy is $t_{2}-t_{1}=\theta_{2}\left(x_{1}^{*}, 21\right)$, with

$$
\theta_{2}\left(x_{1}^{*}, 21\right)=\frac{1}{2 \rho} \ln \left[\left(1-\rho \beta_{2} x_{2}^{*}\right) \frac{x_{1}^{*}}{x_{2}^{*}}+\rho \beta_{2} x_{2}^{*}\right]=\frac{1}{2 \rho} \ln \left[\frac{\Phi_{i}\left(x_{1}^{*}, 21\right)}{\Phi_{i}\left(x_{2}^{*}, 21\right)}\right] .
$$

- In regime 11: extraction strategies satisfy

$$
\begin{aligned}
& \gamma_{1}^{1} \Phi_{1}(x, 11)=\gamma_{2}^{1} \Phi_{2}(x, 11)=\Lambda\left(x_{1}^{*}, x_{2}^{*}\right)+\rho x, \\
& \text { with } \Lambda\left(x_{1}^{*}, x_{2}^{*}\right)=\frac{\Gamma\left(x_{2}^{*}\right)+\rho x_{1}^{*}\left[1-\zeta\left(x_{1}^{*} ; x_{2}^{*}\right)\right]}{\zeta\left(x_{1}^{*} ; x_{2}^{*}\right)}
\end{aligned}
$$

The level of the stock for switching $x_{1}^{*}$ is uniquely defined by

$$
\rho \omega_{1}\left(x_{1}^{*}\right)=e^{-\rho \theta_{2}\left(x_{1}^{*}, 21\right)} \ln \left(1-\rho \beta_{2} x_{2}^{*}\right)+\ln \left[\zeta\left(x_{1}^{*} ; x_{2}^{*}\right)\right]-\ln \left(\frac{\gamma_{1}^{2}}{\gamma_{1}^{1}}\right) .
$$

With $\left(x_{1}^{*}, x_{2}^{*}\right)$ being determined above, the switching time $t_{1}=\theta_{1}\left(x_{0}, 11\right)$ is

$$
\theta_{1}\left(x_{0}, 11\right)=\frac{1}{2 \rho} \ln \left(\frac{\Lambda\left(x_{1}^{*}, x_{2}^{*}\right)+\rho x_{0}}{\Lambda\left(x_{1}^{*}, x_{2}^{*}\right)+\rho x_{1}^{*}}\right)=\frac{1}{2 \rho} \ln \left[\frac{\Phi_{i}\left(x_{0}, 11\right)}{\Phi_{i}\left(x_{1}^{*}, 11\right)}\right] .
$$

Let us start with the analysis of extraction strategies. One immediately observes that, in each regime, the two players have the same extraction rates, but generally

\footnotetext{
8 These technical conditions are not central in the upcoming economic analysis.
} 
not the same consumption rates. This feature is due to the logarithmic utility. From equations (9) and (10), we see that if $\beta_{2}>0$, then the adoption of the new technology by player 2 translates into a downward jump in the extraction rate at time $t_{2}$. Intuitively, with the new technology, one needs less resource to produce a given amount of the consumption good. The impact of player 2's adoption on her own consumption from time $t_{2}$ onward must be positive (for otherwise, she would not make the switch).

Even more interesting is the fact that it is unsure whether the downward adjustment in extraction also occurs at player 1's switching date. Indeed, the movement of the extraction rate at $t_{1}$ depends on whether $\zeta\left(x_{1}^{*} ; x_{2}^{*}\right) \lessgtr 1,{ }^{9}$ which is unclear in general. This emphasizes the impact of the interaction via switching strategies on extraction strategies. Player 1 knows that he will be worse off after player 2's/adoption because he will bear the decrease in extraction rates while being unable to compensate it by changing his own technology, as it is fixed after time $t_{1}$. This may induce him to compensate this future anticipated costly event by increasing the extraction rate at $t_{1}$.

As an illustration, we can take a numerical example, ${ }^{10}$ for which the extraction rate actually increases at the date of adoption of player, 1. As depicted in Figure 1, the upward jump in the extraction rate allows player 1 to substantially increase the rate of consumption at $t_{1}$ in the anticipation of future bad times.

As to the switching strategies, the combination of the optimality conditions displayed in Theorem 1 ((1) for player 2, and (2) for player 1) yields conditions (11) and (13) in the application. According to (11), for instance, player 2 chooses the switching point $x_{2}^{*}$ that equalizes the net marginal gain of adoption (RHS) to the (constant value) direct marginal switching cost, $\rho \omega_{2}\left(x_{2}^{*}\right)$ (LHS). The gain from adoption is measured in terms of increased consumption rates; it is given by the difference between the direct marginal gain from adoption, $-\ln \left(\frac{\gamma_{2}^{2}}{\gamma_{2}^{1}}\right)$, and the downward adjustment of the extraction rate, represented by $\ln \left(1-\rho \beta_{2} x_{2}^{*}\right)<0 .{ }^{11}$

\footnotetext{
9 This expression provides information on the magnitude and direction of the adjustment of extraction - and player 2's consumption since she keeps using the same technology - occurring at the instant of the transition from regime 11 to regime 21.

10 We use the following set of parameters: $x_{0}=1500, \beta_{1}=0.001, \beta_{2}=0.01, \chi_{1}=1, \chi_{2}=10$, $\gamma_{1}^{1}=2, \gamma_{1}^{2}=1.715, \gamma_{2}^{1}=2, \gamma_{2}^{2}=1, \rho=0.04$. For these parameter values, technological gain from adoption is larger for player 2 than for player 1; but adoption costs are much higher for the former. There exists a unique PCNE, in linear feedback rules for extraction, at which player 1 switches first, then followed by player 2 , switching times and points being given by: $\left(t_{1}, x_{1}^{*}\right)=(9.548,678.332)$ and $\left(t_{2}, x_{2}^{*}\right)=(16.847,352.686)$.

${ }^{11}$ This is evaluated in utility terms. The same logic is at work for player 1 except that the adjustment of the extraction rate at his switching time is given by $\ln \left[\zeta\left(x_{1}^{*} ; x_{2}^{*}\right)\right]$ and he also has to take into account the change in extraction at player 2's adoption time, once discounted appropriately.
} 

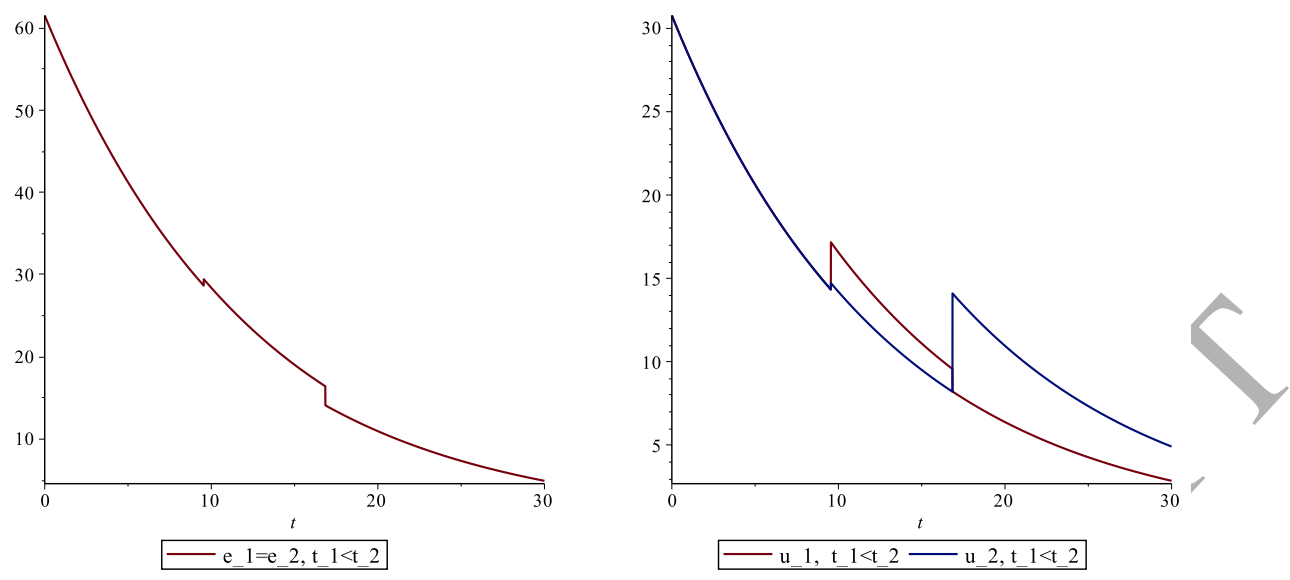

Fig. 1: Evolution of extraction (left) and consumption (right) rates at the PCNE

Moreover, equation (12) confirms that player 2's time-to-go before switching is defined in terms of player 1's switching point, $x_{1}^{*}$, the discount rate and some parameters characterizing regime 21 , that players leave, and regime 22 , that players reach. Hence, player 1 is able to affect player 2's switching strategy and will take this influence into account in the first period problem. Note also that the optimal switching date of player 2 is increasing in $x_{1}^{*}$. The larger the resource stock at which player 1 decides to switch, the later the adoption of player 2. In other words, switching rapidly for player 1 tends to delay the adoption time of player 2 .

This naturally leads us to the next Section that deals with the analysis of the impact of the interaction through switching strategies on the timing of technology adoption.

\subsection{Impact of the interaction through switching strategies}

From the analysis of Section 3.1, player 1's optimality conditions for switching can be written as (by substitution of the functional forms in conditions (2) of Theorem 1):

$$
\begin{aligned}
& \ln \left[\frac{u_{1}^{11}\left(t_{1}\right)}{u_{1}^{21 *}\left(t_{1}\right)}\right]=-\rho \omega_{1}\left(x_{1}^{*}\right)+e^{-\rho \theta_{2}\left(x_{1}^{*}, 21\right)} \ln \left[\frac{u_{1}^{22 *}\left(t_{2}\right)}{u_{1}^{21 *}\left(t_{2}\right)}\right] \\
& {\left[\gamma_{1}^{2} u_{1}^{21 *}\left(t_{1}\right)\right]^{-1}-\left[\gamma_{1}^{1} u_{1}^{11 *}\left(t_{1}\right)\right]^{-1}=\omega_{1}^{\prime}\left(x_{1}^{*}\right)+\theta_{2}^{\prime}\left(x_{1}^{*}, 21\right) e^{-\rho \theta_{2}\left(x_{1}^{*}, 21\right)} \ln \left[\frac{u_{1}^{22 *}\left(t_{2}\right)}{u_{1}^{21 *}\left(t_{2}\right)}\right]}
\end{aligned}
$$

Compared to the single-agent problem, both conditions are modified. The LHS of the first equation in (14) reflects the marginal gain from extending the horizon of the first regime. If there exists $0<t_{1}<t_{2}$ then this marginal gain must be equal to the 
marginal cost of switching at $t_{1}$. Now, the marginal switching cost (RHS) is augmented (in absolute magnitude) by the extra-term $e^{-\rho \theta_{2}\left(x_{1}^{*}, 21\right)} \ln \left[\frac{u_{1}^{22 *}\left(t_{2}\right)}{u_{1}^{21 *}\left(t_{2}\right)}\right]$. Player 1 anticipates that his switching decision will be followed by the switch (in finite time too) of the second player and that player 2's switch will be costly to him. So, it means that player 1 's marginal cost of switching at time $t_{1}$ is higher than it would be in the absence of player 2. Other things equal ( $x_{1}$ constant), this would imply that the switch should occur at a later date, i.e., player 1 , when interacting with player 2 , has anincentive to postpone adoption.

The second equation in (14) equalizes the marginal benefit from an extra unit of the state variable $x_{1}$ (LHS) with the corresponding marginal cost (RHS). This cost is lower in the game than in the control problem because, from $(12), \theta_{2}^{\prime}\left(x_{1}^{*}, 21\right)>0$ and we know that $\ln \left[\frac{u_{1}^{22 *}\left(t_{2}\right)}{u_{1}^{21 *}\left(t_{2}\right)}\right]<0$. Indeed, changing $x_{1}$ marginally yields an additional benefit here. Other things equal ( $t_{1}$ constant), it allows player 1 to induce player 2 to delay the instant of her switch. The impact of player 2's switch will then be felt less acutely because of discounting. This in turn implies that player 1's adoption should occur at a higher $x_{1}^{*}$. This second effect tends to make it worthwhile for player 1 to adopt at an earlier date (because the trajectory of $x$ is monotone non-increasing).

In summary, as a result of the interaction with player 2, player 1 has an incentive to delay the adoption of the new technology (first-order effect corresponding to the first condition in (14)). It does not mean, however, that he will not adopt before player 2. According to the second condition in (14), the sooner the adoption of player 1 , the lower the negative impact of player 2's adoption on his welfare (second-order effect).

To conclude this analysis, a striking result can be obtained by focusing on the special case where $\omega_{1}(.) \equiv 0$ : Player 1's switching cost is identically zero, so that it is independent of the stock of resource. In this case, if there were no game-theoretic considerations, we know that the solution of the optimal control problem would be $t_{1}=0$ : Player 1 would adopt the new technology instantaneously because it is more efficient than the old one. Conclusions are very different in our game setting. It is optimal for player 1 to switch at a strictly positive date $t_{1}$ because, by delaying adoption, player 1 affects player 2's decision in such a way that the cost imposed by her adoption is dampened. In the same vein, from the numerical example, we obtain that if player 1 was the only one allowed to switch technology, then he would adopt at an earlier date than when he has to adapt to player 2's adoption $\left(t_{1}^{\prime}=0.805<t_{1}=9.548\right)$.

Let us now turn to the last part of the application, whose purpose is to examine paraître, à paraître. DOI : 10.1016/j.jedc.2017.01.008 
the conditions under which the timing under scrutiny is robust to deviations in players' switching strategies, and players do not opt for corner strategies.

\subsection{Incentives to deviate from the specified timing}

As outlined in Section 3.2, a player may find the timing $0 \leqq t_{1}<t_{2} \leqq \infty$ non-optimal. For instance, guessing that player 1 will switch at $t_{1}(<\infty)$, player 2 may prefer switching at a date no later than $t_{1}$, i.e., deviate from the specified timing. As far as non-optimal timing are concerned, it can be shown that

Proposition 2. Suppose player 1 adopts the new technology at $t_{1}$. If player 2 wants to deviate from the timing $0 \leqq t_{1}<t_{2} \leqq \infty$, then it must hold that

$$
\rho \omega_{2}\left(x_{1}^{*}\right) \leq \ln \left(1-\rho \beta_{2} x_{1}^{*}\right)-\ln \left(\frac{\gamma_{2}^{2}}{\gamma_{2}^{1}}\right)
$$

Given that player 2's adoption takes place at $t_{2}$, player 1 has an incentive to deviate only if:

$$
\rho \omega_{1}\left(x_{2}^{*}\right) \geq \ln \left[\zeta\left(x_{2}^{*} ; x_{2}^{*}\right)\right]+\ln \left(1-\rho \beta_{2} x_{2}^{*}\right)-\ln \left(\frac{\gamma_{1}^{2}}{\gamma_{1}^{1}}\right) .
$$

For an interpretation, it is enough to consider player 2's necessary condition, given by equation (15) and to draw a parallel with the corresponding condition for a interior solution (11). The question is when would player 2 find it optimal to skip regime 21 and directly go to regime 22, given that player 1 has adopted at date $0<t_{1}<\infty$ ? It turns out that what matters to player 2 , when she contemplates the opportunity to adopt right after player 1's adoption, is still the balance between the marginal gain and cost of adoption, now evaluated at player 1's switching point. So according to (15), player 2 wants to deviate from the timing $t_{1} \leqq t_{2}$ when the net marginal benefit from switching (RHS) outweighs the direct marginal switching cost (LHS), as soon as player 1 has adopted and for a switching point $x_{1}^{*}$.

As mentioned in the discussion following Theorem 2, necessary conditions for the timing not to be robust to deviations are derived from the analysis of hypothetical corner solutions where the switching time of one player is assumed to be given. ${ }^{12}$ But switching times are not given in the game setting since they are part of players' strategies. In particular, when looking at player 2's problem, condition (15), whose standard

\footnotetext{
${ }^{12}$ For instance, condition (15) is obtained when considering $t_{2} \rightarrow t_{1}$, for $t_{1}$ given; this is the limit of the optimality condition (11) for $x_{2}^{*} \rightarrow x_{1}^{*}$, when one replaces the equality with inequality " $\leq$ ".
} 
interpretation in a single-agent framework would be that player 2 is willing to adopt immediately at $t_{1}$, actually characterizes the situation where she has an incentive to deviate from the considered timing, i.e., to adopt before player 1. A symmetric reasoning applies to player 1 .

As the whole analysis has been conducted for the timing $0 \leqq t_{1}<t_{2} \leqq \infty$, one logically expects that we can set out conditions under which the timing is indeed robust to deviations. This is done by imposing, for $i=1,2$ :

$$
\begin{aligned}
& \rho \omega_{2}\left(x_{i}^{*}\right)+\ln \left(\frac{\gamma_{2}^{2}}{\gamma_{2}^{1}}\right)>\rho \omega_{1}\left(x_{i}^{*}\right)+\ln \left(\frac{\gamma_{1}^{2}}{\gamma_{1}^{1}}\right), \\
& \zeta\left(x_{i}^{*} ; x_{i}^{*}\right)>1
\end{aligned}
$$

which are sufficient for the opposite of condition (15) and (16) hold. Condition (17a) is especially intuitive as it requires the first player to adopt be also the one who faces the lowest net adoption cost, $\rho \omega_{i}\left(x_{i}^{*}\right)+\ln \left(\frac{\gamma_{i}^{2}}{\gamma_{i}^{1}}\right)$, regardless of the switching point considered.

Before ending the analysis, it is worth considering the other situations where the PCNE associated with timing $0 \leqq t_{1}<t_{2} \leqq \infty$ may exhibit a true corner structure with, for example, player 1 adopting immediately at $t_{1}=0$, or player 2 never adopting $\left(t_{2}=\infty\right)$. Our results, that are obtained by applying the conditions (3)-(5) of Theorem 2 and (6) to the resource game, can be summarized in the following proposition.

Proposition 3. - Assume that player 1's switching problem has an interior solution $t_{1}$. A sufficient condition for player 2 to choose the "never switching strategy," so that $0<t_{1}<t_{2}=\infty$, is that

$$
\rho \omega_{2}(0)+\ln \left(\frac{\gamma_{2}^{2}}{\gamma_{2}^{1}}\right) \geq 0
$$

- Assume that player 2's switching problem has an interior solution $t_{2}$. A necessary condition for player 1 to switch immediately at the beginning, so that $0=t_{1}<$ $t_{2}<\infty$ is

$$
\rho \omega_{1}\left(x_{0}\right)+\ln \left(\frac{\gamma_{1}^{2}}{\gamma_{1}^{1}}\right) \leq e^{-\rho \theta_{2}\left(x_{0}, 21\right)} \ln \left(1-\beta_{2} \rho x_{2}^{*}\right)+\ln \left[\zeta\left(x_{0} ; x_{2}^{*}\right)\right]
$$

- A combination of immediate and never switching $0=t_{1}<t_{2}=\infty$ may arise only 
if (18) and

$$
\rho \omega_{1}\left(x_{0}\right)+\ln \left(\frac{\gamma_{1}^{2}}{\gamma_{1}^{1}}\right) \leq 0
$$

hold.

Conditions for a corner solution also have very simple interpretations. For instance, according to condition (18), player 2 never finds it worthwhile to adopt the new technology if the fixed cost of adoption $\rho \omega_{2}(0)$, weighted by the rate of time preference, is larger than the direct gain from switching even when the resource gets close to exhaustion (in our setting, the stock of resource is asymptotically exhausted). In the same vein, for a player to be willing to adopt the new technology immediately it must hold that the switching cost at the initial resource level is lower than the gain from adoption. In the latter case, the particular tradeoff is influenced by the other player's switching decision to switch in finite time (19) or to keep the old technology forever (20). ${ }^{13}$

Since we have been interested so far in the analysis of the interior solution, we finally have to impose conditions that allow us to disregard corner solutions. This we can do by assuming that

$$
\begin{aligned}
& \rho \omega_{2}(0)+\ln \left(\frac{\gamma_{2}^{2}}{\gamma_{2}^{1}}\right)<0, \\
& \rho \omega_{1}\left(x_{0}\right)+\ln \left(\frac{\gamma_{1}^{2}}{\gamma_{1}^{1}}\right)>\max \left\{0, e^{-\rho \theta_{2}\left(x_{0}, 21\right)} \ln \left(1-\beta_{2} \rho x_{2}^{*}\right)+\ln \left[\zeta\left(x_{0} ; x_{2}^{*}\right)\right]\right\} .
\end{aligned}
$$

The last important thing to note is that conditions (17) and (21) are also sufficient to conclude that the PCNE with $0<t_{1}<t_{2}<\infty$, discussed in Section 4.1, indeed exists.

\section{Discussion}

\subsection{Further insights from numerical analysis}

It is worth assessing the impact of technology adoption in the resource game. For that purpose, we can compare the PCNE with $0<t_{1}<t_{2}<\infty$ with the benchmark situation in which none of the players can take a regime change decision. In this case, there also exists a unique PCNE, which mimics the corner solution with $t_{1}=t_{2}=\infty$.

\footnotetext{
13 There are three cases left: (i) Players might wish to adopt their new technology at the same date and for the same stock of resource. Or, (ii) they might both prefer switching instantaneously; or (iii) on the contrary they might prefer sticking to the first technology forever. If there is heterogeneity in switching costs, case (i) cannot be an equilibrium outcome. The conditions for having the two other possibilities can easily be derived from Proposition 3 (see the Appendix B.4).
} 
But one should keep in mind that, for the parameter values chosen (see footnote 12), this benchmark cannot arise as an equilibrium outcome in the game with two players having a switching decision.

The comparison between the PCNE with technology adoption and the one without allows to highlight the impact of the interaction through switching strategies on the extraction and consumption rates. As depicted in Figure 2 (left), the opportunity to adopt a new - more efficient - technology tends to ramp up extraction due to higher extraction rates in regime 11 and 21, followed by lower extraction rates in 22. Indeed, the slightly faster pace of exhaustion of the resource (see Figure 2, right) implies that the extraction rate must be lower in the last regime (in both cases, the common extraction rate is equal to $\rho x$ ). Overall, as depicted in Figure 2 (center), players' consumption rates are higher than the ones they will enjoyed in the absence of adoption of better technologies.
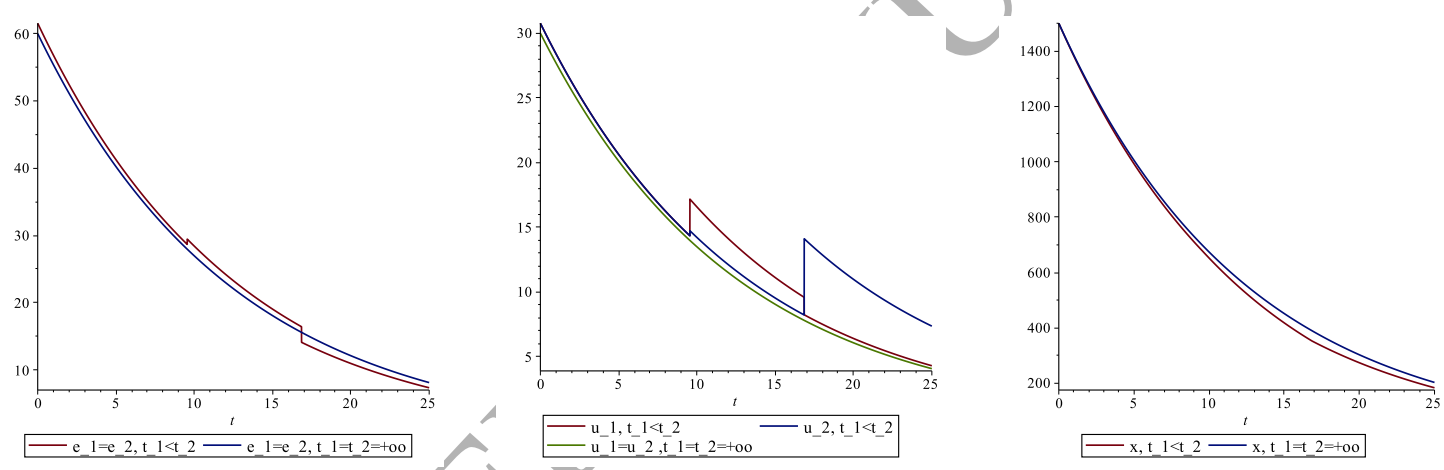

Fig. 2: Extraction and consumption rates at the PCNE with and without adoption

Propositions 1-3 have emphasized the role of technology parameters on one hand, and cost parameters on the other hand, in the determination of the equilibrium timing. We can further benefit from the numerical example by performing a brief comparative statics exercise. Changing for instance the technology parameters in the numerical example (the baseline scenario being given in footnote 12) has the following consequences. Increasing $\gamma_{1}^{2}$ slightly $\left(\gamma_{1}^{2^{\prime}}=1.72>\gamma_{1}^{2}=1.715\right)$ makes technology adoption less rushed for player 1 as the technological gain is smaller. So adoption by player 1 occurs later and for a lower stock of resource $\left(t_{1}^{\prime}=11.093>t_{1}=9.548\right)$. This in turn implies that adoption by player 2 is slightly delayed due to strategic interaction $\left(t_{2}^{\prime}=16.913>t_{2}=16.847\right)$. A larger increase in $\gamma_{1}^{2}\left(\gamma_{1}^{2^{\prime}}=1.75>\gamma_{1}^{2}=1.715\right)$ has a stronger impact on the equilibrium because player 1 now wants to deviate from the 
timing $t_{1}<t_{2}$ by switching in second position. On the contrary, a sufficient decrease in $\gamma_{1}^{2}\left(\gamma_{1}^{2^{\prime}}=1.6<\gamma_{1}^{2}=1.715\right)$ means that technology adoption is even more worthwhile and gives player 1 the incentive to adopt a corner strategy by choosing $t_{1}^{\prime}=0$. In the same vein, a rise in $\gamma_{2}^{2}\left(\gamma_{2}^{2^{\prime}}=1.2>\gamma_{2}^{2}=1\right)$, which means that switching technology is less beneficial to player 2 , tends to postpone her adoption $\left(t_{2}^{\prime}=29.241>t_{2}=16.847\right)$. At the same time, and in accordance with player 2's adjustment, player 1's adoption occurs earlier and for a higher stock of the resource $\left(t_{1}^{\prime}=1.811>t_{1}=9.548\right)$. Another interesting feature is that as player 2's adoption is now less harmful to player 1 (the downward jump in extraction is smaller and $t_{2}$ larger), he no longer has to compensate this future costly event by increasing the extraction rate at his own date of adoption (see the discussion in Section 4.1). Thus, extraction rates jump down at both switching times. Finally, if player 2' gain from adoption becomes higher because of a smaller $\gamma_{2}^{2}$ $\left(\gamma_{2}^{2^{\prime}}=0.8<\gamma_{2}^{2}=1\right)$, then we obtain that none of the players find the timing optimal anymore. Both want to switch positions and allow player 2 to move first.

\subsection{Other potential applications of the methodology}

In the present analysis, we have chosen the simplest economic problem on purpose as we wanted to illustrate how the methodology works in an application. However, our contribution can be useful for researchers working in various fields of the economic analysis. It provides economists with a powerful tool to assess any situation where the decision maker can take some "discrete" decisions, as opposed to standard continuous controls, that affect his/her situation and, since we do not live in an isolated world, the situation of other decision makers surrounding him/her. This may include much more involved economic problems such as the following ones. ${ }^{14}$

Our methodology can first be applied to extended versions of recent papers in resource economics, like Jaakola (2012) (or Gerlagh and Liski, 2011). Jaakola considers the dynamic and strategic interaction between an oil exporter, who owns an exhaustible polluting resource, and an oil importer who invests in R\&D to make a backstop technology cheaper. In this paper, one of the players, the oil importer, has a regime switching or timing decision as he/she chooses the instant when to switch to the backstop (so the date of economic exhaustion of the resource). ${ }^{15}$ In order for this general problem

\footnotetext{
${ }^{14}$ A richer list of relevant applications and more institution-oriented examples can be found in Boucekkine et al. (2013).

${ }^{15}$ Note that Jaakola mostly focuses on the open-loop Nash equilibrium while we believe that commitment requirements are simply too strong to model switching strategies as open-loop strategies.
} 
to provide an interesting application of our theory, we would have to assign a regime switching decision to the other player. It is well-known that a major weakness of oil exporting economies is the lack of economic diversification, which notably makes them vulnerable to the volatilities of prices (Arezki et al., 2011). One option would then be to assume that the oil-exporter can also decide, given the threat of a switch to the backstop by the other player, when to engage in a process of diversification. Still on natural resources management by oligopolies, another interesting analysis is the one of the impact of oil discovery on the oligopoly equilibrium (Benchekroun and Long, 2006). The problem would consist in defining oil discovery as a timing strategy, players being able to increase theirs stocks at some date $t_{i}$ by paying a lumpy exploration cost defined over the actual stocks of resources of all players. This would also contribute to the literature on the trade-offs between exploration and extraction, which usually disentangles those issues by assuming that an oil producer chooses its exploration effort at the initial date and then its extraction path (the problem being solved backward, see Gaudet and Lasserre, 1988, and more recently, Daubanes and Lasserre, 2015).

The second class of applications of our theory has to do with the dynamics of institutions in resource-rich economies. For instance, Boucekkine et al. (2016) analyze the dynamic and strategic interaction between an autocratic elite and the citizens. In their setting, there is an initial regime during which the elite control all the resources and take all the decisions. The citizens can only take a regime change decision by choosing the date of a costly revolution against the elite, which leads the system to a more democratic regime. A natural extension of this work may consist in allowing the elite to have a switching strategy too. In line with this paper, the elite may be able to revise their redistribution and/or repression policy at some point in time, when the threat of revolution becomes serious. A related but different topic is the one dealing with conflicts related to natural resources. Van der Ploeg (2016) develops a differential game in which a resource extraction problem is coupled with a contest for the control of the resource by rival groups. He adopts the traditional way of modeling conflict by using a contest success function (see Tullock, 1980). His approach basically boils down to disentangling the problem of choosing the fighting efforts - which becomes static and whose resolution gives the symmetric equilibrium in these efforts at each date from the problem of choosing extraction rates - which is in essence a dynamic one. An alternative approach would be to assume that rival groups can decide the date when to trigger (at some cost) a conflict against the others to expropriate them or increase the share of the stock of resource it controls. This is actually very much in line with Tornell paraître, à paraître. DOI : 10.1016/j.jedc.2017.01.008 
(1997)'s game in which players can change the system of property rights defined over a resource.

One last particularly interesting application is related to climate change. Following the climate negotiations in Paris (COP21, Dec. 2015), one may consider the situation of different countries, or groups of countries (North versus South typically) that may be initially out of a binding international agreement (like the Kyoto Protocol) but may decide to join it, or remain out forever, by balancing the costs and benefits of each alternative. And it goes without saying that the timing decision of each group (when to reach the agreement) has strong repercussions on the other groups through the impact on the concentration of GHG, or the penalties (trade barriers) that might be imposed to groups that prefer to remain out and then run the risk of being accused of environmental dumping.

\section{Conclusion}

This paper develops a general two-player differential game with regime switching strategies. The interaction between players is assumed to be governed by two kinds of strategies. At each point in time, they have to choose an action that influences the evolution of a state variable. In addition, they may decide on the switching time between alternative and consecutive regimes. We pay attention to the piecewise closed-loop Nash equilibrium: the switching strategy is defined as a function of the state of the system. Compared to the standard optimal control problem with regime switching, necessary optimality conditions are modified only for the first player to switch. When choosing the switching strategy, this player must take into account that (i) his decision will influence the other player's strategy, and (ii) the other player's switch will affect his welfare. Furthermore, we have exhibited and interpreted the conditions characterizing the timing at the piecewise closed-loop equilibrium, i.e., the timing that is robust to deviations in switching strategies.

In the second part of this paper, we applied this new theoretical framework to solve a game of exhaustible resource extraction with technological regime switching. It was assumed that, at a given cost, players have the option to adopt a more efficient extraction technology. We then obtained sufficient conditions guaranteeing that both players switch in finite time. Moreover, we investigated the impact of this new source of interaction on the adoption strategy of the first-player-to-switch. There is an interplay between two conflicting effects. On the one hand, adoption by the second-player-to- 
switch imposes a cost to the one who switches first because it implies a drop in his future consumption that starts from her adoption time. Thus, the first-player-to-switch may have an incentive to delay adoption. On the other hand, because of discounting, delaying the switch of the other player will allow the first-player-to-switch to incur a lower indirect cost, in present-value terms. This cost-saving effect of delaying is smaller, the higher is the discount rate. This tends to mitigate the incentive to delay adoption.

Overall, the methodology presented in this paper may pave the way to handre a wider class of problems in economics. Potential extensions include the analysis of technology adoption in a climate change game, the consideration of the interaction between the elites and the citizens in a game of institutional regime changes (Acemoglu and Robinson, 2006), and the analysis of conflict between rival groups for the management of natural resources (van der Ploeg and Rohner, 2012). These issues will be addressed in future research endeavors. 


\section{References}

[1] Acemoglu, D. and Robinson, J.: Economic Origins of Dictatorship and Democracy, Cambridge Books, Cambridge University Press (2006).

[2] Amir, R.: Continuous Stochastic Games of Capital Accumulation with Convex Transitions. Games and Economic Behavior 15, 111-131 (1996).

[3] Amit, R.: Petroleum reservoir exploitation: Switching from primary to secondary recovery. Operations Research 34, 534-549 (1986).

[4] Arezki, R., Gylfason, T., and Sy, A.: Beyond the Curse: Policies to Harness the Power of Natural Resources. International Monetary Fund (2011).

[5] Benchekroun, H. and Long, N.V.: The curse of windfall gains in a non-renewable resource oligopoly. Australian Economic Papers 45, 99-105 (2006).

[6] Boucekkine, R., Saglam, C. and Vallée, T.: Technology adoption under embodiment: a two-stage optimal control approach. Macroeconomic Dynamics 8, 250-271 (2004).

[7] Boucekkine, R., Pommeret, A. and Prieur, F.: Optimal regime switching and threshold effects. Journal of Economie Dynamics and Control 37, 2979-2997 (2013).

[8] Boucekkine, R., Prieur, F. and Puzon, K.: On the timing of political regime changes in resource-dependent economies. European Economic Review 85, 188207 (2016).

[9] Boucekkine, R., Krawczyk, J. and Vallée T.: Environmental quality versus economic performance: A dynamic game approach. Optimal Control Applications and Methods 32, 29-46 (2011).

[10] Daubanes, J., and Lasserre, P.: Optimum Commodity Taxation with a NonRenewable Resource. CESIfo working paper \#5270 (2015).

[11] Davis, M.: Markov Models \& Optimization. In Chapman and Hall/CRC; 1st edition (August 1, 1993).

[12] Dockner, E., Long, N. V., and Sorger, G.: Analysis of Nash Equilibria in a Class of Capital Accumulation Games. Journal of Economic Dynamics and Control 20, 1209-1235 (1996). 
[13] Dutta, P. K. and Sundaram, R.: The Tragedies of the Commons. Economic Theory 3, 413-426 (1993).

[14] Fudenberg, D Tirole, J.: Preemption and rent equalization in the adoption of new technology. Review of Economic Studies, 52(3), 383-401 (1985).

[15] Gaudet, G., and Lasserre, P.: On Comparing Monopoly and Competition in Exhaustible Resource Exploitation. Journal of Environmental Economics and Management 15, 412-418 (1988).

[16] Gerlagh, R. and Liski, M.: Strategic Resource Dependence, Journal of Economic Theory 146, 699-727 (2011).

[17] Haurie, A., Krawczyk, J. and Zaccour, G.: Games and Dynamic Games, NOW Publisher (2012).

[18] Haurie, A. and Moresino, F.: Computation of S-adapted equilibria in piecewise deterministic games via stochastic programming methods. In : Altman, Eitan Ed. Advances in Dynamic Games and Applications. Boston : Birkhäuser, p. 225-252. Annals of the International Society of Dynamic Games; vol. 6 (2001).

[19] Jaakola, N.: Green Technologies and the Protracted End to the Age of Oil: A strategic analysis. OxCarre research paper \#99 (2012).

[20] Lemoine, D. and Traeger, C.) (2014). Watch Your Step: Optimal Policy in a Tipping Climate. American Economic Journal: Economic Policy 6, 137-166

[21] Long, NV.: A Survey Of Dynamic Games In Economics. World Scientific Publishing Co. Pte. Ltd. (2010).

[22] Long, NV.: Dynamic Games in the Economics of Natural Resources: A Survey. Dynamic Games and Applications 1(1), 115-148 (2011).

[23] Makris, M.: Necessary conditions for infinite horizon discounted two-stage optimal control problems. Journal of Economic Dynamics and Control 25, 1935-1950 (2001).

[24] Reinganum, JF.: On the diffusion of new technology: A game theoretic approach. Review of Economic Studies 48, 395-405 (1981). 
[25] Tahvonen, O and Withagen, C.: Optimality of irreversible pollution accumulation. Journal of Economic Dynamics and Control 20, 1775-1795 (1996).

[26] Tomiyama, K.: Two-stage optimal control problems and optimality conditions. Journal of Economic Dynamics and Control 9, 317-337 (1985).

[27] Tornell, A.: Economic growth and decline with endogenous property rights. Journal of Economic Growth, 2(3), 219-50, (1997).

[28] Tullock, G.: Efficient rent-seeking. In J. M. Buchanan, R. D. Tollison, \& G. Tullock (Eds.), Toward a theory of the rent-seeking society (pp. 97-112) College Station: Texas A. \& M. University Press (1980).

[29] Valente, S.: Endogenous growth, backstop technology adoption, and optimal jumps. Macroeconomic Dynamics 15, 293-325 (2011).

[30] Van der Ploeg, F.: Political economy of dynamic resource wars. Oxcarre working paper \#97, revised (2016).

[31] van der Ploeg, F. and Rohner, D.: Wâr and natural resource exploitation, European Economic Review, 56(8), 1714-1729 (2012).

[32] van der Ploeg, F. and de Zeeuw, A.: Non-Cooperative and Cooperative Responses to Climate Catastrophes in the Global Economy: A North-South Perspective. OxCarre research paper \#149 (2014).

[33] Zemel, A.: Adaptation,mitigation and risk: An analytic approach. Journal of Economic Dynamics and Control 51, 133-147 (2015).

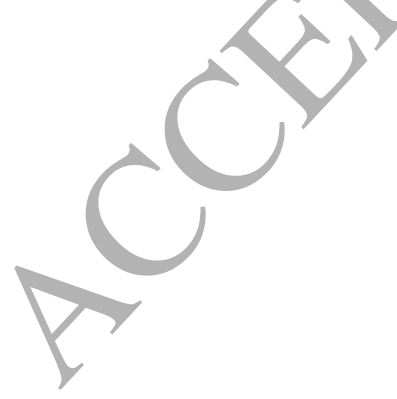




\section{Appendix}

\section{A Theory}

\section{A.1 Proof of Theorems 1\&2}

Let the triplet $\left(u_{1}^{*}(t), u_{2}^{*}(t), x^{*}(t)\right)$ be the path followed by each player's strategy and the stock variable when players adopt markovian rules for the action strategies, for every $t \in[0,+\infty)$. A restriction of this path to $\left[t_{j-1}, t_{j}\right]$, which corresponds to a particular regime say $s, j=1,2,3$ with $t_{0}=0$ and $t_{3}=\infty$, continues to characterize the solution of the subgame with $x^{*}\left(t_{j}-1\right)=x_{j-1}, t_{j-1}$ and $t_{j}$ given and with the maximization of $\int_{t_{j-1}}^{t_{j}} F_{i}^{s}\left(u_{1}, u_{2}, x\right) e^{-\rho t} d t$ as player $i$ 's objective, $i=1,2$.

The proof proceeds in two steps. We start by constructing the first order variation of the value function resulting from one player's small deviation from the equilibrium path. Hereafter, we shall go through the main steps of the proof because our approach extensively relies on Amit (1986) and we refer the reader to this paper for more details. We focus on the timing $0 \leqq t_{1} \leqq t_{2} \leqq \infty$, i.e., on the case where player 1 is the first to switch, followed by player 2. Second, since Amit considers an optimal control problem, we put forward the specificity of the game setting and provide the corresponding new necessary conditions for switching. Necessary optimality conditions for the other timing $0 \leqq t_{2} \leqq t_{1} \leqq \infty$ can be obtained by symmetry. For notational convenience, we do not make the dependence of decision rules on the regime explicit. If $t_{1}$ is player 1 switching time then from the definition of the switching rule, we have $t_{2}=t_{1}+\theta_{2}\left(x_{1}^{*}(t)\right)$. The strategy $\theta_{2}\left(x_{1}^{*}\left(t_{1}\right)\right)$ is not a feedback rule in the usual sense as it does not depend on any value of the stock $x(t)$ but only on the level $x^{*}\left(t_{1}\right)$ at which player 2 's switching problem starts. That is why we introduce the new concept of piecewise deterministic Nash equilibrium (PCNE), which conveys the idea that there nevertheless exists some sort of feedback/effect in the switching rules that operates through the specific level of the stock variable, $x^{*}\left(t_{1}\right)$.

Player 1's payoffs evaluated along the PCNE, the strategies of player 2 being given, can be-written as:

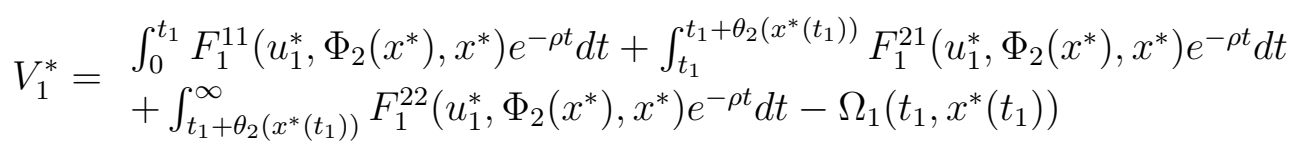

Assume that player 1 considers the opportunity to change his switching time by a small 
amount $\delta t_{1}$. Let $\left(u_{1}(t), u_{2}(t), x(t)\right)$ be the vector of feasible controls and state associated with this change. Player 1 's switching time becomes $t_{1}+\delta t_{1}$ whereas the one of player 2 (as anticipated by player 1 ) is now given by $t+\delta t_{1}+\theta_{2}(x(t))$ for $t \geq t_{1}+\delta t_{1}$. Then, player 1's payoffs become:

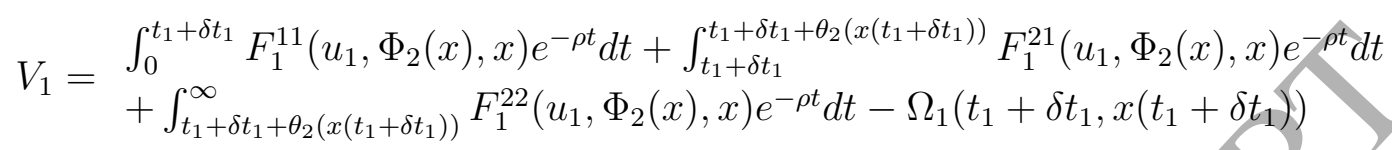

In order to compute the variation, we consider the following decompositions:

$$
\begin{gathered}
\int_{0}^{t_{1}+\delta t_{1}} F_{1}^{11}\left(u_{1}, \Phi_{2}(x), x\right) e^{-\rho t} d t= \\
\int_{0}^{t_{1}} F_{1}^{11}\left(u_{1}, \Phi_{2}(x), x\right) e^{-\rho t} d t+\int_{t_{1}}^{t_{1}+\delta t_{1}} F_{1}^{11}\left(\widehat{u}_{1}, \Phi_{2}(x), x\right) e^{-\rho t} d t, \\
\int_{t_{1}+\delta t_{1}}^{t_{1}+\delta t_{1}+\theta_{2}\left(x\left(t_{1}+\delta t_{1}\right)\right)} F_{1}^{21}\left(u_{1}, \Phi_{2}(x), x\right) e^{-\rho t} d t=\int_{t_{1}}^{t_{1}+\theta_{2}\left(x^{*}\left(t_{1}\right)\right.} F_{1}^{21}\left(u_{1}, \Phi_{2}(x), x\right) e^{-\rho t} d t \\
-\int_{t_{1}}^{t_{1}+\delta t_{1}} F_{1}^{21}\left(u_{1}, \Phi_{2}(x), x\right) e^{-\rho t} d t+\int_{t_{1}+\theta_{2}\left(x^{*}\left(t_{1}\right)\right.}^{t_{1}+\delta t_{1}+\theta_{2}\left(x\left(t_{1}+\delta t_{1}\right)\right)} F_{1}^{21}\left(u_{1}, \Phi_{2}(x), x\right) e^{-\rho t} d t,
\end{gathered}
$$

and

$$
\begin{gathered}
\int_{\left.t_{1}+\delta t_{1}+\theta_{2}(x)\left(t_{1}+\delta t_{1}\right)\right)}^{\infty} F_{1}^{22}\left(u_{1}, \Phi_{2}(x), x\right) e^{-\rho t} d t= \\
\int_{t_{1}+\theta_{2}\left(x^{*}\left(t_{1}\right)\right.}^{\infty} F_{1}^{22}\left(u_{1}, \Phi_{2}(x), x\right) e^{-\rho t} d t-\int_{t_{1}+\theta_{2}\left(x^{*}\left(t_{1}\right)\right.}^{t_{1}+\delta t_{1}+\theta_{2}\left(x\left(t_{1}+\delta t_{1}\right)\right)} F_{1}^{22}\left(u_{1}, \Phi_{2}(x), x\right) e^{-\rho t} d t .
\end{gathered}
$$

Rearranging the terms and introducing the notations $F_{1}^{s}\left(u_{1}, \Phi_{2}(x), x\right)=G_{1}^{s}\left(u_{1}, x\right)$, $G_{1}^{s *}=G_{1}^{s}\left(u_{1}^{*}, x^{*}\right), f^{s}\left(u_{1}, \Phi_{2}(x), x\right)=g^{s}\left(u_{1}, x\right)$, and $g^{s *}=g^{s}\left(u_{1}^{*}, x^{*}\right)$, the variation of player 1 's payoffs $\delta V_{1}=V_{1}-V_{1}^{*}$ is equal to:

$$
\begin{aligned}
& \int_{0}^{t_{1}}\left\{\left[G_{1}^{11}\left(u_{1}, x\right) e^{-\rho t}+\lambda_{1}^{11} g^{11}\left(u_{1}, x\right)\right]-\left[G_{1}^{11}\left(u_{1}^{*}, x^{*}\right) e^{-\rho t}+\lambda_{1}^{11} g^{11}\left(u_{1}^{*}, x^{*}\right)\right]-\lambda_{1}^{11} \dot{h}^{11}\right\} d t \\
& +\int_{t_{1}}^{t_{1}+\theta_{2}\left(x^{*}\left(t_{1}\right)\right)}\left\{\left[G_{1}^{21}\left(u_{1}, x\right) e^{-\rho t}+\lambda_{1}^{21} g^{21}\left(u_{1}, x\right)\right]-\left[G_{1}^{21}\left(u_{1}^{*}, x^{*}\right) e^{-\rho t}+\lambda_{1}^{21} g^{21}\left(u_{1}^{*}, x^{*}\right)\right]-\lambda_{1}^{21} \dot{h}^{21}\right\} d t \\
& +\int_{t_{1}+\theta_{2}\left(x^{*}\left(t_{1}\right)\right)}^{\infty}\left\{\left[G_{1}^{22}\left(u_{1}, x\right) e^{-\rho t}+\lambda_{1}^{22} g^{22}\left(u_{1}, x\right)\right]-\left[G_{1}^{22}\left(u_{1}^{*}, x^{*}\right) e^{-\rho t}+\lambda_{1}^{22} g^{22}\left(u_{1}^{*}, x^{*}\right)\right]-\lambda_{1}^{22} \dot{h}^{22}\right\} d t \\
& +\int_{t_{1}+\delta t_{1}}^{t_{1}}\left[G_{1}^{11}\left(u_{1}, x\right)-G_{1}^{21}\left(u_{1}, x\right)\right] e^{-\rho t} d t+\int_{t_{1}+\theta_{2}\left(x^{*}\left(t_{1}\right)\right)}^{\left.t_{1}\right)}\left[G_{1}^{21}\left(u_{1}, x\right)-G_{1}^{22}\left(u_{1}, x\right)\right] e^{-\rho t} d t \\
& -\Omega_{1}\left(t_{1}+\delta t_{1}, x\left(t_{1}+\delta t_{1}\right)\right)+\Omega_{1}\left(t_{1}, x^{*}\left(t_{1}\right)\right)
\end{aligned}
$$


where we have added to the integrand of the first three lines the term $\lambda_{1}^{s}\left(g^{s}\left(u_{1}, x\right)-\right.$ $\dot{x})-\lambda_{1}^{s}\left(g^{s}\left(u_{1}^{*}, x^{*}\right)-\dot{x}^{*}\right)$, for any differentiable functions $\lambda_{1}^{s}, s=11,21,22$, and defined, in any regime $s$, the deviation $h^{s}$ as $h^{s}=x-x^{*}$.

Integrating by parts the terms $\int-\lambda_{1}^{s} \dot{h}^{s} d t$ and using appropriate boundary - including the initial and the transversality - conditions yield: $\delta V_{1}=$

$$
\begin{aligned}
& \int_{0}^{t_{1}}\left\{\left[H_{1}^{11}\left(t, u_{1}, x\right)-H_{1}^{11 *}(t)\right]+\dot{\lambda}_{1}^{11} h^{11}\right\} d t+\int_{t_{1}}^{t_{1}+\theta_{2}\left(x^{*}\left(t_{1}\right)\right)}\left\{\left[H_{1}^{21}\left(t, u_{1}, x\right)-H_{1}^{21 *}(t)\right]+\dot{\lambda}_{1}^{21} h^{21}\right\} d t \\
& +\int_{t_{1}+\theta_{2}\left(x^{*}\left(t_{1}\right)\right)}^{\infty}\left\{\left[H_{1}^{22}\left(t, u_{1}, x\right)-H_{1}^{22 *}(t)\right]+\dot{\lambda}_{1}^{11} h^{11}\right\} d t+\int_{t_{1}}^{t_{1}+\delta t_{1}}\left[G_{1}^{11}\left(u_{1}, x\right)-G_{1}^{21}\left(u_{1}, x\right)\right] e^{-\rho t} d t \\
& +\int_{\left.t_{1}+\delta t_{1}+\theta_{2}(x)\left(t_{1}+\delta t_{1}\right)\right)}^{\left.t^{*}\left(t_{1}\right)\right)}\left[G_{1}^{21}\left(u_{1}, x\right)-G_{1}^{22}\left(u_{1}, x\right)\right] e^{-\rho t} d t-\Omega_{1}\left(t_{1}+\delta t_{1}, x\left(t_{1}+\delta t_{1}\right)\right)+\Omega_{1}\left(t_{1}, x^{*}\left(t_{1}\right)\right) \\
& -\lambda_{1}^{11}\left(t_{1}\right) h^{11}\left(t_{1}\right)+\lambda_{1}^{21}\left(t_{1}\right) h^{21}\left(t_{1}\right)-\lambda_{1}^{21}\left(t_{2}\right) h^{21}\left(t_{2}\right)+\lambda_{1}^{22}\left(t_{2}\right) h^{22}\left(t_{2}\right)
\end{aligned}
$$

where $t_{2}=t_{1}+\theta_{2}\left(x^{*}\left(t_{1}\right)\right), H_{1}^{s}\left(t, u_{1}, x\right)=G_{1}^{s}\left(u_{1}, x\right) e^{-\rho t}+\lambda_{1}^{s} g^{11}\left(u_{1}, x\right)$ is regime $s$ Hamiltonian and $H_{1}^{s *}(t)$ is the same Hamiltonian evaluated along the equilibrium trajectory.

Now we want to obtain a linear approximation of $\delta V_{1}$. For $\delta t_{1}$ close to zero and $h^{s}$, $\delta u_{1}=u_{1}-u_{1}^{*}$ small, we first compute the following first order Taylor series:

$$
H_{1}^{s}\left(t, u_{1}, x\right) \simeq H_{1}^{s *}(t)+\frac{\partial H_{1}^{s *}(t)}{\partial u_{1}} \delta u_{1}+\frac{\partial H_{1}^{s *}(t)}{\partial x} h^{s}
$$

and,

$$
\begin{aligned}
& \int_{t_{1}+\delta t_{1}}^{t_{1}}\left[G_{1}^{11}\left(u_{1}, x\right)-G_{1}^{21}\left(u_{1}, x\right)\right] e^{-\rho t} d t \simeq\left[G_{1}^{11 *}-G_{1}^{21 *}\right] e^{-\rho t_{1}} \delta t_{1} \\
& \int_{t_{1}+\theta_{2}\left(x^{*}\left(t_{1}\right)\right)}^{\left.\left.t_{1}+\delta t_{1}\right)\right)}\left[G_{1}^{21}\left(u_{1}, x\right)-G_{1}^{22}\left(u_{1}, x\right)\right] e^{-\rho t} d t \simeq \\
& {\left[G_{1}^{21 *}-G_{1}^{22 *}\right] e^{-\rho\left(t_{1}+\theta_{2}\left(x^{*}\left(t_{1}\right)\right)\right)}\left(\delta t_{1}+\theta_{2}\left(x\left(t_{1}+\delta t_{1}\right)\right)-\theta_{2}\left(x^{*}\left(t_{1}\right)\right)\right)}
\end{aligned}
$$

Making use of these approximations, (22) can be rewritten as:

$$
\begin{aligned}
& \int_{0}^{t_{1}}\left\{\frac{\partial H_{1}^{11 *}(t)}{\partial u_{1}} \delta u_{1}+\left[\frac{\partial H_{1}^{11 *}(t)}{\partial x}+\dot{\lambda}_{1}^{11}\right] h^{11}\right\} d t+\int_{t_{1}}^{t_{1}+\theta_{2}\left(x^{*}\left(t_{1}\right)\right)}\left\{\frac{\partial H_{1}^{21 *}(t)}{\partial u_{1}} \delta u_{1}+\left[\frac{\partial H_{1}^{21 *}(t)}{\partial x}+\dot{\lambda}_{1}^{21}\right] h^{21}\right\} d t \\
& +\int_{t_{1}+\theta_{2}\left(x^{*}\left(t_{1}\right)\right)}^{\infty}\left\{\frac{\partial H_{1}^{2 *}(t)}{\partial u_{1}} \delta u_{1}+\left[\frac{\partial H_{1}^{22 *}(t)}{\partial x}+\dot{\lambda}_{1}^{22}\right] h^{22}\right\} d t+\left[G_{1}^{11 *}-G_{1}^{21 *}\right] e^{-\rho t_{1}} \delta t_{1} \\
& +\left[G_{1}^{21 *}-G_{1}^{22 *}\right] e^{-\rho\left(t_{1}+\theta_{2}\left(x^{*}\left(t_{1}\right)\right)\right.}\left(\delta t_{1}+\theta_{2}\left(x\left(t_{1}+\delta t_{1}\right)\right)-\theta_{2}\left(x^{*}\left(t_{1}\right)\right)\right) \\
& -\Omega_{1}\left(t_{1}+\delta t_{1}, x\left(t_{1}+\delta t_{1}\right)\right)+\Omega_{1}\left(t_{1}, x^{*}\left(t_{1}\right)\right) \\
& -\lambda_{1}^{11}\left(t_{1}\right) h^{11}\left(t_{1}\right)+\lambda_{1}^{21}\left(t_{1}\right) h^{21}\left(t_{1}\right)-\lambda_{1}^{21}\left(t_{2}\right) h^{21}\left(t_{2}\right)+\lambda_{1}^{22}\left(t_{2}\right) h^{22}\left(t_{2}\right)
\end{aligned}
$$

Next we take the linear parts of the following Taylor expansions:

$$
\begin{aligned}
& \theta_{2}\left(x\left(t_{1}+\delta t_{1}\right)\right) \simeq \theta_{2}\left(x^{*}\left(t_{1}\right)\right)+\theta_{2}^{\prime}\left(x^{*}\left(t_{1}\right)\right) \delta x_{1} \\
& \Omega_{1}\left(t_{1}+\delta t_{1}, x\left(t_{1}+\delta t_{1}\right)\right) \simeq \Omega_{1}\left(t_{1}, x^{*}\left(t_{1}\right)\right)+\frac{\partial \Omega_{1}\left(t_{1}, x^{*}\left(t_{1}\right)\right)}{\partial t_{1}} \delta t_{1}+\frac{\partial \Omega_{1}\left(t_{1}, x^{*}\left(t_{1}\right)\right)}{\partial x_{1}} \delta x_{1}
\end{aligned}
$$


with $\delta x_{1}=x\left(t_{1}+\delta t_{1}\right)-x^{*}\left(t_{1}\right)$ the difference between the value taken by the state variable at the new switching time $t_{1}+\delta t_{1}$ and the equilibrium value $x^{*}\left(t_{1}\right)$. Again, let's consider the following approximation:

$$
x\left(t_{1}+\delta t_{1}\right) \simeq x\left(t_{1}\right)+\dot{x}^{*}\left(t_{1}\right) \delta t_{1}
$$

where $\dot{x}\left(t_{1}\right)$ has been replaced with $\dot{x}^{*}\left(t_{1}\right)$, which is possible if $x\left(t_{1}\right)$ is close enough to $x^{*}\left(t_{1}\right)$, i.e., if our functional forms are smooth enough (see Assumption 1). We also need to use the same difference of state values at player 2's switching times, $\delta x_{2}=$ $x\left(t_{1}+\delta t_{1}+\theta_{2}\left(x\left(t_{1}+\delta t_{1}\right)\right)-x^{*}\left(t_{1}+\theta_{2}\left(x^{*}\left(t_{1}\right)\right)\right.\right.$, together with:

$x\left(t_{1}+\delta t_{1}+\theta_{2}\left(x\left(t_{1}+\delta t_{1}\right)\right) \simeq x\left(t_{1}+\theta_{2}\left(x^{*}\left(t_{1}\right)\right)+\dot{x}^{*}\left(t_{1}+\theta_{2}\left(x^{*}\left(t_{1}\right)\right)\left(\delta t_{1}+\theta_{2}^{\prime}\left(x^{*}\left(t_{1}\right)\right) \delta x_{1}\right)\right.\right.\right.$

Observing that $\dot{x}^{*}$ in any regime $s$ is equal to $g^{s *}$, the deviation $h^{s}$ can be expressed in terms of the variations $\delta t_{1}, \delta x_{1}$ and $\delta x_{2}$ :

$$
\begin{aligned}
& h^{s}\left(t_{1}\right)=\delta x_{1}-g^{s *} \delta t_{1} \text { for } s=11,21, \\
& h^{s}\left(t_{2}\right)=\delta x_{2}-g^{s *}\left(\delta t_{1}+\theta_{2}^{\prime}\left(x^{*}\left(t_{1}\right)\right) \delta x_{1}\right) \text { for } s=21,22 .
\end{aligned}
$$

Putting all these elements together allows us to get the expression of $\delta V_{1}$. Assuming that Pontryagin conditions

$$
\begin{aligned}
& \frac{\partial H_{1}^{s *}(t)}{\partial u_{1}}=0 \\
& \dot{\lambda}_{1}^{s}=-\frac{\partial H_{1}^{s *}(t)}{\partial x}
\end{aligned}
$$

are met in every regime $s=11,21,22$, we obtain:

$$
\begin{aligned}
& {\left[H_{1}^{11 *}\left(t_{1}\right)-H_{1}^{21 *}\left(t_{1}\right)+H_{1}^{21 *}\left(t_{2}\right)-H_{1}^{22 *}\left(t_{2}\right)-\frac{\partial \Omega_{1}\left(t_{1}, x^{*}\left(t_{1}\right)\right)}{\partial t_{1}}\right] \delta t_{1} } \\
&\left.\delta V_{1}=\begin{array}{l}
{ }^{2} \\
+
\end{array} \lambda_{1}^{21}\left(t_{1}\right)-\lambda_{1}^{11}\left(t_{1}\right)+\theta_{2}^{\prime}\left(x^{*}\left(t_{1}\right)\right)\left(H_{1}^{21 *}\left(t_{2}\right)-H_{1}^{22 *}\left(t_{2}\right)\right)-\frac{\partial \Omega_{1}\left(t_{1}, x^{*}\left(t_{1}\right)\right)}{\partial x_{1}}\right] \delta x_{1} \\
&+
\end{aligned}
$$

For the trajectory $\left(u_{1}^{*}(t), u_{2}^{*}(t), x^{*}(t)\right)$, with switching times $t_{1}$ and $t_{2}$, to be optimal for player 1 we must have $\delta V_{1} \leq 0$. Then, from (25), we can move a step forward in the characterization of the necessary optimality conditions by observing that:

If $\delta t_{1}, \delta x_{1}$ and $\delta x_{2}$ are completely free and independent variables, then it must hold 
that:

$$
\begin{aligned}
& H_{1}^{11 *}\left(t_{1}\right)-\frac{\partial \Omega_{1}\left(t_{1}, x^{*}\left(t_{1}\right)\right)}{\partial t_{1}}=H_{1}^{21 *}\left(t_{1}\right)-\left[H_{1}^{21 *}\left(t_{2}\right)-H_{1}^{22 *}\left(t_{2}\right)\right] \\
& \lambda_{1}^{11}\left(t_{1}\right)+\frac{\partial \Omega_{1}\left(t_{1}, x^{*}\left(t_{1}\right)\right)}{\partial x_{1}}=\lambda_{1}^{21}\left(t_{1}\right)+\theta_{2}^{\prime}\left(x^{*}\left(t_{1}\right)\right)\left[H_{1}^{21 *}\left(t_{2}\right)-H_{1}^{22 *}\left(t_{2}\right)\right] \\
& \lambda_{1}^{21}\left(t_{2}\right)=\lambda_{1}^{22}\left(t_{2}\right)
\end{aligned}
$$

The same methodology can be displayed to analyze player 2's switching problem. The analysis is actually simpler when we solve her problem because by definition of the switching rule, any small deviation $\delta t_{2}$ by player 2 from the switching time $t_{2}$ has no impact on player 1's switching strategy when the timing is $0 \leqq t_{1} \leqq t_{2} \leqq \infty$. This means that the first subgame still runs from the initial time $t_{0}=0$ to the switching time $t_{1}=\theta_{1}\left(x_{0}\right)$ after a deviation. Player 2 switching problem is very much the same as an optimal control problem. So we can skip all the technical details and directly present the expression of the variation $\delta V_{2}=V_{2}-V_{2}^{*}$ :

$$
\begin{aligned}
& \delta V_{2}=\left[H_{2}^{21 *}\left(t_{2}\right)-H_{2}^{22 *}\left(t_{2}\right)-\frac{\partial \Omega_{2}\left(t_{2}, x^{*}\left(t_{2}\right)\right)}{\partial t_{2}}\right] \delta t_{2} \\
& +\left[\lambda_{2}^{22}\left(t_{2}\right)-\lambda_{2}^{21}\left(t_{2}\right)-\frac{\partial \Omega_{2}\left(t_{2}, x^{*}\left(t_{2}\right)\right)}{\partial x_{2}}\right] \delta x_{2}+\left[\lambda_{2}^{21}\left(t_{1}\right)-\lambda_{2}^{11}\left(t_{1}\right)\right] \delta x_{1} .
\end{aligned}
$$

The equivalent of conditions (26), for player 2, are then given by:

$$
\begin{aligned}
& H_{2}^{21 *}\left(t_{2}\right)-\frac{\partial \Omega_{2}\left(t_{2}, x^{*}\left(t_{2}\right)\right)}{\partial t_{2}}=H_{2}^{22 *}\left(t_{2}\right) \\
& \lambda_{2}^{21}\left(t_{2}\right)+\frac{\partial \Omega_{2}\left(t_{2}, x^{*}\left(t_{2}\right)\right)}{\partial x_{2}}=\lambda_{2}^{22}\left(t_{2}\right) \\
& \lambda_{2}^{11}\left(t_{1}\right)=\lambda_{2}^{21}\left(t_{1}\right)
\end{aligned}
$$

This might seem to be the end of the proof of Theorem 1, (26) and (28) characterizing the interior solution $0<t_{1}<t_{2}$. But it is not since we have now to account for the specificity of the game structure. In particular, we need to understand whether $\delta x_{j}$ is a completely free and independent variable for player $i, i, j=1,2, i \neq j$, when she/he contemplates the impact of a deviation from her/his switching strategy. To show that $\delta x_{2}$ is in fact necessarily equal to 0 from player 1 's perspective, we focus on the optimization program faced by each player in regime 21 and we work by contradiction (the same reasoning applies to show that $\delta x_{1}=0$ for player 2). Assume that we have solved the subgame corresponding to regime 22 and obtained the value function $V_{i}\left(t_{2}, x\left(t_{2}\right)\right), i=1,2$. Then, working backward, we have the following considerations.

Player 1, guessing that player 2's strategy is $u_{2}=\Phi_{2}(x)$ (here we do not make the guess in the switching strategy explicit since it plays no role in the subsequent analysis), 
solves

$$
\max _{\left\{u_{1}\right\}} \int_{t_{1}}^{t_{2}} F_{1}^{21}\left(u_{1}, \Phi_{2}(x), x\right) e^{-\rho t} d t+V_{1}\left(t_{2}, x\left(t_{2}\right)\right)
$$

s.t.

$$
\begin{aligned}
& \dot{x}=f^{21}\left(u_{1}, \Phi_{2}(x), x\right) \\
& x\left(t_{1}\right)=x_{1} \text { given and } x\left(t_{2}\right)=x_{2} \text { free. }
\end{aligned}
$$

Player 2 also decides when to switch to regime 22 and thus solves:

$$
\max _{\left\{u_{2}\right\}, t_{2}} \int_{t_{1}}^{t_{2}} F_{2}^{21}\left(\Phi_{1}(x), u_{2}, x\right) e^{-\rho t} d t+V_{2}\left(t_{2}, x\left(t_{2}\right)\right)-\Omega\left(t_{2}, x\left(t_{2}\right)\right)
$$

s.t.

$$
\begin{aligned}
& \dot{x}=f^{21}\left(\Phi_{1}(x), u_{2}, x\right) \\
& x\left(t_{1}\right)=x_{1} \text { given and } x\left(t_{2}\right)=x_{2} \text { free. }
\end{aligned}
$$

The critical point, in the formulation above, is the assumption that even if it is player 2 that has to take her regime change decision, $x\left(t_{2}\right)$ is considered free by both players. If it is the case, then from the third condition in (26) and the second in (28), we must have:

$$
\begin{aligned}
& \lambda_{1}^{21}\left(t_{2}\right)=\lambda_{1}^{22}\left(t_{2}\right) \\
& \lambda_{2}^{21}\left(t_{2}\right)+\frac{\partial \Omega_{2}\left(t_{2}, x^{*}\left(t_{2}\right)\right)}{\partial x_{2}}=\lambda_{2}^{22}\left(t_{2}\right) .
\end{aligned}
$$

Now assume that there is a (unique) pair of markovian strategies $\left(\Phi_{1}(x), \Phi_{2}(x)\right)$, that solves the Pontryagin conditions in any regime. Then, in particular, we have for $i=1,2$ and $s=21,22$ :

$$
\frac{\partial F_{i}^{s}\left(\Phi_{1}(x), \Phi_{2}(x), x\right)}{\partial u_{i}}+\frac{\partial f^{s}\left(\Phi_{1}(x), \Phi_{2}(x), x\right)}{\partial u_{i}} \lambda_{i}^{s}=0
$$

As long as the problem is well behaved (functional forms are twice continuously differentiable, i.e., Assumption 1 holds), strategies $\Phi_{i}(x)$ are continuous functions of $x$, and (30) simply defines the costate variable as a continuous function of the state (of course, the denominator below must be different from 0 ):

$$
\lambda_{i}^{s}=-\frac{\frac{\partial F_{i}^{s}\left(\Phi_{1}(x), \Phi_{2}(x), x\right)}{\partial u_{i}}}{\frac{\partial f^{s}\left(\Phi_{1}(x), \Phi_{2}(x), x\right)}{\partial u_{i}}} \equiv \Psi_{i}^{s}(x)
$$

For simplicity assume that functions $\Psi_{i}(x)$ are also monotonic (the proof easily extends to the non-monotonic case), then they are invertible and we obtain a continuous 
relationship linking the two costate variables:

$$
\lambda_{2}^{s}=\Psi_{2}^{s}\left(\left(\Psi_{1}^{s}\right)^{-1}\left(\lambda_{1}^{s}\right)\right) \equiv \Pi^{s}\left(\lambda_{1}^{s}\right)
$$

that holds for $s=21,22$. This implies that

$$
\lambda_{2}^{21}\left(t_{2}\right)=\Pi^{21}\left(\lambda_{1}^{21}\left(t_{2}\right)\right) \text { and } \lambda_{2}^{22}\left(t_{2}\right)=\Pi^{22}\left(\lambda_{1}^{22}\left(t_{2}\right)\right),
$$

which cannot hold together with switching conditions $(29)$ as long as $\Pi^{21}(.) \neq \Pi^{22}($.$) ;$ this is where the contradiction arises.

In sum, the analysis above shows that in most well-behaved (tractable) differential games, there exists a (continuous) relationship between players' co-state variables at the PCNE and at any instant, including the switching times. Thus, the conditions involving the co-states in (26) and (28) cannot hold all together because they imply that one co-state is continuous whereas the other jumps at each switching time. This means that the variation $\delta x_{2}$ in (25) and $\delta x_{1}$ in (27) must be set equal to zero, i.e., player $i$ deviation $\delta t_{i}$ has no impact of level of the state variable at player $j$ ' switching time $x\left(t_{j}\right)$. Therefore, the necessary condítions for an interior solution $0<t_{1}<t_{2}<\infty$ reduce to

$$
\begin{aligned}
& H_{1}^{11 *}\left(t_{1}\right)-\frac{\partial \Omega_{1}\left(t_{1}, x^{*}\left(t_{1}\right)\right)}{\partial t_{1}}=H_{1}^{21 *}\left(t_{1}\right)-\left[H_{1}^{21 *}\left(t_{2}\right)-H_{1}^{22 *}\left(t_{2}\right)\right] \\
& \lambda_{1}^{11}\left(t_{1}\right)+\frac{\partial \Omega_{1}\left(t_{1}, x^{*}\left(t_{1}\right)\right)}{\partial x}=\lambda_{1}^{21}\left(t_{1}\right)+\theta_{2}^{\prime}\left(x^{*}\left(t_{1}\right)\right)\left[H_{1}^{21 *}\left(t_{2}\right)-H_{1}^{22 *}\left(t_{2}\right)\right]
\end{aligned}
$$

and,

$$
\begin{aligned}
& H_{2}^{21 *}\left(t_{2}\right)-\frac{\partial \Omega_{2}\left(t_{2}, x^{*}\left(t_{2}\right)\right)}{\partial t_{2}}=H_{2}^{22 *}\left(t_{2}\right) \\
& \lambda_{2}^{21}\left(t_{2}\right)+\frac{\partial \Omega_{2}\left(t_{2}, x^{*}\left(t_{2}\right)\right)}{\partial x_{2}}=\lambda_{2}^{22}\left(t_{2}\right)
\end{aligned}
$$

Now we move to the necessary optimality conditions for corner solutions.

Let us start yith player 1's conditions assuming that player 2 switches at $t_{2} \in(0, \infty)$. If feasible variations are only of the type $\delta t_{1} \geq 0$, which corresponds to the solution $t_{1}=0$, then the first necessary condition in (34) is replaced with (the second still holds):

$$
H_{1}^{11 *}\left(t_{1}\right)-\frac{\partial \Omega_{1}\left(t_{1}, x^{*}\left(t_{1}\right)\right)}{\partial t_{1}} \leq H_{1}^{21 *}\left(t_{1}\right)-\left[H_{1}^{21 *}\left(t_{2}\right)-H_{1}^{22 *}\left(t_{2}\right)\right] \text { if } 0=t_{1}<t_{2}
$$

If we rather consider deviations $\delta t_{1} \leq 0$, which means that we focus on the corner 
solution $t_{1}=t_{2}$, then the inequality in (37) has to be reversed, which gives:

$$
H_{1}^{11 *}\left(t_{1}\right)-\frac{\partial \Omega_{1}\left(t_{1}, x^{*}\left(t_{1}\right)\right)}{\partial t_{1}} \geq H_{1}^{21 *}\left(t_{1}\right)-\left[H_{1}^{21 *}\left(t_{2}\right)-H_{1}^{22 *}\left(t_{2}\right)\right] \text { if } t_{1}=t_{2}
$$

As for player 2 , looking at the corner solution $t_{1}=t_{2}$, with the switching time of player 1 positive and finite $t_{1} \in(0, \infty)$, boils down to considering only variations of the type $\delta t_{2} \geq 0$. Then, the first condition in (35) becomes (the second one is still valid):

$$
H_{2}^{21 *}\left(t_{2}\right)-\frac{\partial \Omega_{2}\left(t_{2}, x^{*}\left(t_{2}\right)\right)}{\partial t_{2}} \leq H_{2}^{22 *}\left(t_{2}\right) \text { if } t_{1}=t_{2}
$$

Finally, there is no necessary condition for the corner solution $t_{2}=\infty$ (see Makris, 2001). However, a sufficient condition for this case is

$$
\left.H_{2}^{21 *}\left(t_{2}\right)-\frac{\partial \Omega_{2}\left(t_{2}, x^{*}\left(t_{2}\right)\right)}{\partial t_{2}}>H_{2}^{22 *}\left(t_{2}\right) \text { (for any } t_{1} \leq t_{2}<\infty\right)
$$

This completes the proof of Theorems $1 \& 2$.

\section{B Application}

We restrict attention to linear feedback strategies: $\Phi_{j}(x, s)=a_{j}^{s}+b_{j}^{s} x$. In any regime $s$, player $i$ 's present value Hamiltonian is given by:

$$
H_{i}^{s}=\ln \left(u_{i}^{s}\right) e^{-\rho t}-\lambda_{i}^{s}\left(\gamma_{i}^{l} u_{i}^{s}+\gamma_{j}^{k}\left(a_{j}^{s}+b_{j}^{s} x\right)\right)
$$

The FOCs are:

$$
\begin{aligned}
& \left(u_{i}^{s}\right)^{-1} e^{-\rho t}=\gamma_{i}^{l} \lambda_{i}^{s} \\
& \dot{\lambda}_{i}^{s}=\gamma_{j}^{k} b_{j}^{s} \lambda_{i}^{s} \\
& \dot{x}=-\gamma_{i}^{l} u_{i}^{s}-\gamma_{j}^{k}\left(a_{j}^{s}+b_{j}^{s} x\right)
\end{aligned}
$$

and have to be combined with the appropriate transversality condition, which depends on whether the regime is terminal, or not. Solving (40), it can easily be checked that players extraction strategies are the same, whatever the regime:

$$
\gamma_{i}^{l} \Phi_{i}(x, s)=\gamma_{j}^{k} \Phi_{j}(x, s)
$$

and, when regime $s$ is terminal, we obtain: $\gamma_{1}^{l} \Phi_{1}(x, s)=\gamma_{2}^{k} \Phi_{2}(x, s)=\rho x$. This property means that players' co-state variables are identical. Moreover, (40)-(41) imply that 
regime $s$ Hamiltonian reduces to $H_{i}^{s}=\left(\ln \left(u_{i}^{s}\right)-2\right) e^{-\rho t}$.

\section{B.1 Player 2's switching problem}

\section{B.1.1 Interior solution (proof of Proposition 1, first item)}

Switching conditions: Assume player 1 has switched at some $t_{1} \in(0, \infty)$, for a switching point $x_{1}$. Using (40), (41) and noticing that $s=22$ is the terminal regime, conditions (1a) and (1b) of Theorem 1 for an interior solution $\left(t_{2}, x_{2}\right)$, are given by

$$
\begin{aligned}
& \ln \left(u_{2}^{21}\left(t_{2}\right)\right)+\rho \omega_{2}\left(x_{2}\right)=\ln \left(\frac{\rho x_{2}}{\gamma_{2}^{2}}\right) \\
& u_{2}^{21}\left(t_{2}\right)=\frac{\rho x_{2}}{\gamma_{2}^{1}\left(1-\beta_{2} \rho x_{2}\right)} .
\end{aligned}
$$

In addition, the consumption strategies in regime 21 are:

$$
\gamma_{1}^{2} \Phi_{1}(x, 21)=\gamma_{2}^{1} \Phi_{2}(x, 21)=\frac{\rho^{2} \beta_{2}\left(x_{2}\right)^{2}}{1-\beta_{2} \rho x_{2}}+\rho x=\Gamma\left(x_{2}\right)+\rho x
$$

From (40)-(42b) and (43), (42a) can be rewritten as

$$
\rho \omega_{2}\left(x_{2}\right)+\ln \left(\frac{\gamma_{2}^{2}}{\gamma_{2}^{1}}\right)=\ln \left(1-\beta_{2} \rho x_{2}\right) .
$$

This equation defines the optimal level for switching, $x_{2}^{*}$, which is indeed independent on the switching time of player 1 .

Characterization of the solution: The RHS of (44) is defined for all $x_{2} \in\left[0,\left(\rho \beta_{2}\right)^{-1}\right)$, decreasing in $x_{2}$ and varying from zero to $-\infty$ as $x$ goes from zero to $\left(\rho \beta_{2}\right)^{-1}$. The LHS is strictly negative at $x_{2}=0$ iff $\ln \left(\frac{\gamma_{2}^{1}}{\gamma_{2}^{2}}\right)>\rho \omega_{2}(0)$ and, since $\beta_{2}>0$, it is strictly increasing in $x_{2}$. Let's for simplicity assume that $x_{0}<\min \left\{\left(\rho \beta_{1}\right)^{-1},\left(\rho \beta_{2}\right)^{-1}\right\}$. Then, there exists)a unique solution $x_{2}^{*}$ in $\left[0, x_{1}^{*}\right]$ iff:

$$
\begin{aligned}
& \rho \omega_{2}(0)+\ln \left(\frac{\gamma_{2}^{2}}{\gamma_{2}^{1}}\right)<0 \\
& \rho \omega_{2}\left(x_{1}^{*}\right)+\ln \left(\frac{\gamma_{2}^{2}}{\gamma_{2}^{1}}\right)>\ln \left(1-\rho \beta_{2} x_{1}^{*}\right) .
\end{aligned}
$$

Replacing consumptions with the expressions given by (43) in the state equation, and solving the resulting differential equation (with the boundary condition $x\left(t_{1}^{*}\right)=x_{1}^{*}$ ) 
yield the expression of the state variable for any $t \in\left[t_{1}, t_{2}\right]$ :

$$
x^{21 *}(t)=\left[x_{1}^{*}+\frac{\rho \beta_{2}\left(x_{2}^{*}\right)^{2}}{1-\beta_{2} \rho x_{2}^{*}}\right] e^{-2 \rho\left(t-t_{1}\right)}-\frac{\rho \beta_{2}\left(x_{2}^{*}\right)^{2}}{1-\beta_{2} \rho x_{2}^{*}} .
$$

Evaluating this equation at $t_{2}$ and solving for $\theta_{2}=t_{2}-t_{1}$, one obtains

$$
\theta_{2}\left(x_{1}^{*}, 21\right)=\frac{1}{2 \rho} \ln \left[\left(1-\rho \beta_{2} x_{2}^{*}\right) \frac{x_{1}^{*}}{x_{2}^{*}}+\rho \beta_{2} x_{2}^{*}\right]=\frac{1}{2 \rho} \ln \left[\frac{\Phi_{i}\left(x_{1}^{*}, 21\right)}{\Phi_{i}\left(x_{2}^{*}, 21\right)}\right]
$$

which gives the time-to-go (before switching) strategy of player 2 as a function of the equilibrium switching point of player $1, x_{1}^{*}$.

\section{B.1.2 Never switching condition (proof of Proposition 3, first item)}

Still assuming that there exists $t_{1} \in(0, \infty)$, the sufficient condition (6) for a never switching solution $\left(t_{2}=\infty\right)$ is:

$$
\ln \left[u_{2}^{21}\left(t_{2}\right)\right]+\rho \omega_{2}\left(x_{2}\right)>\ln \left[u_{2}^{22}\left(t_{2}\right)\right] \text { for all } t_{1} \leq t_{2}<\infty
$$

From (43) and $\gamma_{2}^{2} u_{2}^{22}(t)=\rho x$, this is equivalent to:

$$
\rho \omega_{2}\left(x_{2}\right)+\ln \left(\frac{\gamma_{2}^{2}}{\gamma_{2}^{1}}\right)>\ln \left(1-\beta_{2} \rho x_{2}\right) .
$$

for all admissible $x_{2}>0$. Now take the limit of both the LHS and the RHS of this equation when $t_{2} \rightarrow \infty$. Observing that $x_{2}=x\left(t_{2}\right) \rightarrow 0$ (the stock of resource is exhausted asymptotically), we obtain that the condition

$$
\rho \omega_{2}(0)+\ln \left(\frac{\gamma_{2}^{2}}{\gamma_{2}^{1}}\right) \geq 0
$$

is sufficient to conclude that player 2 will never adopt. This condition is exactly the opposite of the first condition in (45).

The analysis of the last artificial corner case $\left(t_{1}=t_{2}\right)$ is postponed to Appendix B.3 because it requires player 1's switching problem be examined first. 


\section{B.2 Player 1's switching problem}

\section{B.2.1 Interior solution (proof of Proposition 1, second item)}

Switching conditions: Suppose that player 2's regime switching takes place at some $t_{2} \in(0, \infty)$, with $x\left(t_{2}^{*}\right)=x_{2}^{*}$. Direct manipulations of (40), (41), (43), (46) yields the expression of conditions (2a) and (2b) of Theorem 1 :

$$
\begin{aligned}
& \ln \left(u_{1}^{11}\left(t_{1}\right)\right)+\rho \omega_{1}\left(x_{1}\right)=\ln \left(\frac{\Gamma\left(x_{2}^{*}\right)+\rho x_{1}}{\gamma_{1}^{2}}\right)+e^{-\rho \theta_{2}\left(x_{1}, 21\right)} \ln \left(1-\rho \beta_{2} x_{2}^{*}\right) \\
& \gamma_{1}^{1} u_{1}^{11}\left(t_{1}\right)=\frac{\Gamma\left(x_{2}^{*}\right)+\rho x_{1}}{\zeta\left(x_{1} ; x_{2}^{*}\right)}
\end{aligned}
$$

with $\Gamma($.$) defined in (43) and$

$$
\zeta\left(x_{1} ; x_{2}^{*}\right)=1-\frac{e^{-\rho \theta_{2}\left(x_{1}, 21\right)}}{2} \ln \left(1-\rho \beta_{2} x_{2}^{*}\right)-\beta_{1}\left(\Gamma\left(x_{2}^{*}\right)+\rho x_{1}\right) .
$$

Solving for the markovian consumption strategies in regime 11, one finds

$\gamma_{1}^{1} \Phi_{1}(x, 11)=\gamma_{2}^{1} \Phi_{2}(x, 11)=\Lambda\left(x_{1} ; x_{2}^{*}\right)+\rho x$ with $\Lambda\left(x_{1} ; x_{2}^{*}\right)=\frac{\Gamma\left(x_{2}^{*}\right)+\rho x_{1}\left[1-\zeta\left(x_{1} ; x_{2}^{*}\right)\right]}{\zeta\left(x_{1} ; x_{2}^{*}\right)}$.

Substituting $u_{1}^{11}\left(t_{1}\right)$ with the expression in $(49 \mathrm{~b})$, using $(44)$ and $\gamma_{1}^{2} u_{1}^{21}\left(t_{1}\right)=\Gamma\left(x_{2}^{*}\right)+\rho x_{1}$, the optimality condition (49a) can be rewritten as:

$$
\rho \omega_{1}\left(x_{1}\right)+\ln \left(\frac{\gamma_{1}^{2}}{\gamma_{1}^{1}}\right)=e^{-\rho \theta_{2}\left(x_{1}, 21\right)} \ln \left(1-\rho \beta_{2} x_{2}^{*}\right)+\ln \left[\zeta\left(x_{1} ; x_{2}^{*}\right)\right]
$$

Equation (51) defines player 1's switching point $x_{1}^{*}$, which is independent of $t_{2}$. At the PCNE, it has to be evaluated for $x_{2}, \theta_{2}($.$) defined by (44) and (46).$

Note that there is no reason for player 1's switching point to be the same when $t_{2}<\infty$ than when $t_{2}=\infty$. Indeed, when $t_{2}=\infty$, it can be shown that $x_{1}^{*}$ solves: $\ln \left(\frac{\gamma_{1}^{2}}{\gamma_{1}^{1}}\right)+\rho \omega_{1}\left(x_{1}^{*}\right)=\ln \left(1-\beta_{1} \rho x_{1}^{*}\right)$.

Characterization of the solution: $\zeta\left(x_{1} ; x_{2}^{*}\right)$ is defined over $\left(x_{2}^{*}, x_{0}\right)$ with $\zeta^{\prime}\left(x_{1} ; x_{2}^{*}\right)<$ 0 . Let's assume that $\zeta\left(x_{0} ; x_{2}^{*}\right)>0$, so that the logarithm of $\zeta\left(x_{1} ; x_{2}^{*}\right)$ is defined for all admissible $x_{1}$. The LHS of (51) is always positive and increases with $x_{1}$ on the relevant domain whereas the RHS is non monotone because $\theta_{2}($.$) is increasing in x_{1}$. Therefore, a necessary existence condition is $\zeta\left(x_{2}^{*} ; x_{2}^{*}\right)>1$ (otherwise the RHS is always 
negative). Assuming that $\beta_{2}>2 \beta_{1}$, it is easy to check that $\exists ! \bar{x}_{2}^{*} \in\left(0,\left(\rho \beta_{2}\right)^{-1}\right)$ such that $\zeta\left(x_{2}^{*} ; x_{2}^{*}\right)>1$ for all $x_{2}^{*}<\bar{x}_{2}^{*}$. From now on, we will assume that this technical condition holds. Then necessary and sufficient boundary conditions for the existence of $x_{1}^{*} \in\left(x_{2}^{*}, x_{0}\right)$ solving $(51)$ are:

$$
\begin{aligned}
& \rho \omega_{1}\left(x_{0}\right)+\ln \left(\frac{\gamma_{1}^{2}}{\gamma_{1}^{1}}\right)>e^{-\rho \theta_{2}\left(x_{0}, 21\right)} \ln \left(1-\rho \beta_{2} x_{2}^{*}\right)+\ln \left[\zeta\left(x_{0} ; x_{2}^{*}\right)\right], \\
& \rho \omega_{1}\left(x_{2}^{*}\right)+\ln \left(\frac{\gamma_{1}^{2}}{\gamma_{1}^{1}}\right)<\ln \left(1-\rho \beta_{2} x_{2}^{*}\right)+\ln \left[\zeta\left(x_{2}^{*} ; x_{2}^{*}\right)\right] .
\end{aligned}
$$

Using all the material above, we get the expression of the resource stock for all $t \in\left[0, t_{1}\right]: x^{11}(t)=\left(x_{0}+\frac{\Lambda}{\rho}\right) e^{-2 \rho t}-\frac{\Lambda}{\rho}$. Evaluating this expression at $t_{1}$ we finally obtain:

$$
t_{1}=\theta_{1}\left(x_{0}, 11\right)=\frac{1}{2 \rho} \ln \left(\frac{x_{0}+\frac{\Lambda}{\rho}}{x_{1}^{*}+\frac{\Lambda}{\rho}}\right) .
$$

\section{B.2.2 Immediate switching (proof of Proposition 3, second item)}

Still assuming that player 2's switching problem has a solution $t_{2}$ (with $x_{2}^{*}$ that solves 44 ), if player 1 finds it optimal to switch instantaneously then, according to Theorem 2, conditions (4) must hold. In our application, it is given by:

$$
\ln \left[u_{1}^{11}\left(t_{1}\right)\right]+\rho \omega_{1}\left(x_{1}\right) \leq \ln \left[u_{1}^{21}\left(t_{1}\right)\right]+e^{-\rho \theta_{2}^{*}\left(x_{1}, 21\right)} \ln \left(1-\beta_{2} \rho x_{2}^{*}\right)
$$

Making use of condition (49b), (53) reduces to:

$$
\rho \omega_{1}\left(x_{0}\right)+\ln \left(\frac{\gamma_{1}^{2}}{\gamma_{1}^{1}}\right) \leq e^{-\rho \theta_{2}\left(x_{0}, 21\right)} \ln \left(1-\beta_{2} \rho x_{2}^{*}\right)+\ln \left[\zeta\left(x_{0} ; x_{2}^{*}\right)\right]
$$

which is exactly the opposite of the first condition in (52).

\section{B.3 Robustness to deviations (proof of Proposition 2)}

\section{B.3.1 For player 2}

Consider the situation where player 2 has an incentive to deviate from the timing $t_{1} \leqq t_{2}$, given that player 1's switching problem has an interior solution. Condition (3) of Theorem 2 simplifies to:

$$
\ln \left[u_{2}^{21}\left(t_{2}\right)\right]+\rho \omega_{2}\left(x_{2}^{*}\right) \leq \ln \left[u_{2}^{22}\left(t_{2}\right)\right] \text { if } t_{1}=t_{2}
$$


with $\gamma_{2}^{2} u_{2}^{22}\left(t_{2}\right)=\rho x_{2}^{*}$. Together with $(42 \mathrm{~b})$ and given that $x_{2}^{*}=x_{1}^{*}$ in this limit case, (55) simplifies to:

$$
\rho \omega_{2}\left(x_{1}^{*}\right)+\ln \left(\frac{\gamma_{2}^{2}}{\gamma_{2}^{1}}\right) \leq \ln \left(1-\beta_{2} \rho x_{1}^{*}\right),
$$

which is the exact opposite of the second condition in (45). Moreover, in this hypothetical scenario, equation (51), that defines $x_{1}^{*}$, reduces to

$$
\rho \omega_{1}\left(x_{1}^{*}\right)+\ln \left(\frac{\gamma_{1}^{2}}{\gamma_{1}^{1}}\right)=\ln \left(1-\rho \beta_{2} x_{1}^{*}\right)+\ln \left[\zeta\left(x_{1}^{*} ; x_{1}^{*}\right)\right]
$$

So (56) can finally be rewritten as:

$$
\ln \left[\zeta\left(x_{1}^{*} ; x_{1}^{*}\right)\right] \leq\left\{\rho \omega_{1}\left(x_{1}^{*}\right)+\ln \left(\frac{\gamma_{1}^{2}}{\gamma_{1}^{1}}\right)\right\}-\left\{\rho \omega_{2}\left(x_{1}^{*}\right)+\ln \left(\frac{\gamma_{2}^{2}}{\gamma_{2}^{1}}\right)\right\} .
$$

\section{B.3.2 For player 1}

Assume that player 2's switching problem has an interior solution with $t_{2}$ and $x_{2}^{*}$ given. Applying conditions (2b) and (5), the timing is not robust to deviations in player 1's strategy only if:

$$
\begin{aligned}
& \ln \left[u_{1}^{11}\left(t_{1}\right)\right]+\rho \omega_{1}\left(x_{1}^{*}\right) \geq \ln \left[u_{1}^{21}\left(t_{1}\right)\right]+e^{-\rho \theta_{2}\left(x_{1}^{*}, 21\right)} \ln \left(1-\beta_{2} \rho x_{2}^{*}\right) \text { if } t_{1}=t_{2}, \\
& \gamma_{1}^{1} u_{1}^{11}\left(t_{1}\right)=\frac{\Gamma+\rho x_{1}}{\zeta\left(x_{1} ; x_{2}^{*}\right)}
\end{aligned}
$$

Making use of $x_{1}^{*}=x_{2}^{*}, \theta_{2}^{*}\left(x_{2}^{*}, 21\right)=0$, and $\gamma_{1}^{2} u_{1}^{21}\left(t_{2}^{*}\right)=\gamma_{2}^{1} u_{2}^{21}\left(t_{2}^{*}\right)=\Gamma+\rho x_{2}^{*}$, the first condition in (59) is equivalent to:

$$
\rho \omega_{1}\left(x_{2}^{*}\right)+\ln \left(\frac{\gamma_{1}^{2}}{\gamma_{1}^{1}}\right) \geq \ln \left(1-\beta_{2} \rho x_{2}^{*}\right)+\ln \left[\zeta\left(x_{2}^{*} ; x_{2}^{*}\right)\right]
$$

and note that this condition is the opposite of the second boundary condition for an interior solution in (52). Now, from (44), it can also be written as:

$$
\ln \left[\zeta\left(x_{2}^{*} ; x_{2}^{*}\right)\right] \leq\left\{\rho \omega_{1}\left(x_{2}^{*}\right)+\ln \left(\frac{\gamma_{1}^{2}}{\gamma_{1}^{1}}\right)\right\}-\left\{\rho \omega_{2}\left(x_{2}^{*}\right)+\ln \left(\frac{\gamma_{2}^{2}}{\gamma_{2}^{1}}\right)\right\} .
$$

It has the same general expression as (58), except that the reference point is $x_{2}^{*}$ rather than $x_{1}^{*}$. 


\section{B.4 Remaining cases}

- Immediate and never switching: $0=t_{1}<t_{2}=\infty$. From Appendix B.1.2, we know that (48) is sufficient to have $t_{2}=\infty$. In this case, player 1 compares the (marginal) gain from staying forever in regime 11 to the gain from switching directly to 21 . Given that $\gamma_{1}^{l} u_{1}(0)=\rho x_{0}$ for $l=1,2$, the condition for an immediate switching is: $\rho \omega_{1}\left(x_{0}\right)+\ln \left(\frac{\gamma_{1}^{2}}{\gamma_{1}^{1}}\right) \leq 0$.

- Simultaneous interior switches: $0<t_{1}=t_{2}=t<\infty$. From (40) and (41), we have $\lambda_{1}^{s}=\lambda_{2}^{s}$ in any regime $s$. It is clear that the last switching condition in (7) cannot be simultaneously satisfied for the two players whenever $\omega_{1}^{\prime}(x) \neq \omega_{2}^{\prime}(x)$ for all $x$ (recall that $\omega_{i}^{\prime}(x) e^{-\rho t}=\frac{\partial \Omega_{i}(x, t)}{\partial x}$ for $i=1,2$ ).

- Simultaneous instantaneous switches: $t_{1}=t_{2}=0$ : In this case, it must be true that $\rho \omega_{i}\left(x_{0}\right)+\ln \left(\frac{\gamma_{i}^{2}}{\gamma_{i}^{1}}\right) \leq 0$ for $i=1,2$.

- Never switching for both players: $t_{1}=t_{2}=\infty$. This case occurs when condition (48) holds for the two players. 